\title{
Anatomical and Electrophysiological Clustering of Superficial Medial Entorhinal Cortex Interneurons
}

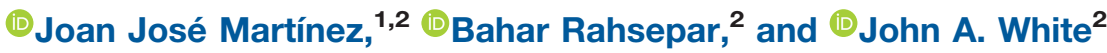

DOI:http://dx.doi.org/10.1523/ENEURO.0263-16.2017

${ }^{1}$ Department of Bioengineering, University of Utah, Salt Lake City, UT 84112 and ${ }^{2}$ Department of Biomedical Engineering, Boston University, Boston, MA 02215

\begin{abstract}
Local GABAergic interneurons regulate the activity of spatially-modulated principal cells in the medial entorhinal cortex (MEC), mediating stellate-to-stellate connectivity and possibly enabling grid formation via recurrent inhibitory circuitry. Despite the important role interneurons seem to play in the MEC cortical circuit, the combination of low cell counts and functional diversity has made systematic electrophysiological studies of these neurons difficult. For these reasons, there remains a paucity of knowledge on the electrophysiological profiles of superficial MEC interneuron populations. Taking advantage of glutamic acid decarboxylase 2 (GAD2)-IRES-tdTomato and PV-tdTomato transgenic mice, we targeted GABAergic interneurons for whole-cell patch-clamp recordings and characterized their passive membrane features, basic input/output properties and action potential (AP) shape. These electrophysiologically characterized cells were then anatomically reconstructed, with emphasis on axonal projections and pial depth. K-means clustering of interneuron anatomical and electrophysiological data optimally classified a population of 106 interneurons into four distinct clusters. The first cluster is comprised of layer 2- and 3 -projecting, slow-firing interneurons. The second cluster is comprised largely of PV + fast-firing interneurons that project mainly to layers 2 and 3. The third cluster contains layer 1- and 2-projecting interneurons, and the fourth cluster is made up of layer 1-projecting horizontal interneurons. These results, among others, will provide greater understanding of the electrophysiological characteristics of MEC interneurons, help guide future in vivo studies, and may aid in uncovering the mechanism of grid field formation.
\end{abstract}

Key words: cluster analysis; entorhinal; excitability; interneuron

\section{Significance Statement}

Despite the critical role that entorhinal inhibitory interneurons play in computation and grid cell formation, the electrophysiological properties of this inhibitory interneuron population remain largely uncharacterized. This study describes systematically the electrophysiology and anatomy of the inhibitory cells in the medial entorhinal cortex (MEC) and introduces a grouping framework for the population. This framework divides the interneuron population into four clusters, based on differences in their axonal projections and electrophysiological properties. These findings confirm and extend findings from previous anatomic studies and will inform future studies of medial entorhinal interneurons.

\section{Introduction}

By modulating the activity of principal neurons, interneurons play a crucial role in the spatial navigation function of the superficial medial entorhinal cortex (MEC;

Received August 31, 2016; accepted September 29, 2017; First published October 6, 2017.

The authors declare no competing financial interests.
Garden et al., 2008; Varga et al., 2010; Beed et al., 2013; Couey et al., 2013; Domnisoru et al., 2013; Pastoll et al., 2013; Buetfering et al., 2014; Fuchs et al., 2016). Among other findings, recent studies have demonstrated that 
MEC GABAergic interneurons mediate stellate-to-stellate cell communication (Couey et al., 2013; Pastoll et al., 2013) and that background inhibition of principal cells is stronger in superficial layers of the MEC than the deeper layers (Woodhall et al., 2005). Grid cell computation work has implemented inhibition-dominated network models to simulate spatial navigation mechanisms (Burak and Fiete, 2009; Pastoll et al., 2013; Thurley et al., 2013). Nevertheless, there is uncertainty as to whether interneurons provide location-dependent input onto grid cells, with Buetfering et al. (2014) providing in vivo data that supports at least parvalbumin (PV)+ interneurons not exhibiting such spatial variability and computational studies of the MEC (Solanka et al., 2015; Shipston-Sharman et al., 2016) contending that other interneuron groups may be providing such a role.

Despite their importance, electrophysiological data for GABAergic interneurons remain scarce (Gloveli et al., 1997; Wolansky et al., 2007; Fuchs et al., 2016). The characterization of superficial MEC interneurons has been difficult for two reasons: the low proportion of interneurons $(\sim 10 \%)$ compared to principal cells (Gatome et al., 2010) and the relative physiologic and anatomic diversity of cortical interneuron populations (Maccaferri and Lacaille, 2003; Whittington and Traub, 2003; Buzsáki et al., 2004; DeFelipe et al., 2013). Previous research suggests that the superficial MEC is anatomically diverse, containing at least seven anatomic categories as defined by soma depth and dendritic morphology (Canto et al., 2008). The anatomic differences in the interneuron population are likely to coincide with different roles within the local cortical circuit (Kepecs and Fishell, 2014). Despite the anatomic categorization of MEC interneurons, the combination of low cell counts and functional diversity has made systematic electrophysiological studies difficult with only a few such studies having been attempted (Gonzalez-Sulser et al., 2014; Fuchs et al., 2016; Ferrante et al., 2017). Limited data are available on the firing pattern of basket cells and chandelier cells, both of which have generally lumped together using their common molecular identifier PV (Wouterlood et al., 1995), but these data do not include passive membrane features, basic input/output measures or action potential (AP) characterization. Furthermore, the electrophysiological properties of remaining cell types in the superficial MEC have remained largely unknown (Gloveli et al., 1997; Wolansky et al., 2007).

This work was supported by National Institutes of Health Grants R01 MH058074 and R01 EB164017 (to J.A.W.).

Acknowledgements: We thank Dr. Mark A. Kramer for helpful discussions regarding clustering methods, Dr. Matt Wachowiak for microscope use, and Dr. Alessandra Angelucci for help with cell reconstruction methods. Dr. Fernando R. Fernandez helped with experimental techniques, advised on data analysis, and provided useful critiques of this manuscript.

Correspondence should be addressed to John A. White, Department of Biomedical Engineering, Boston University, 44 Cummington Mall, Room 403, Boston, MA 02215, E-mail: jwhite@bu.edu.

DOI:http://dx.doi.org/10.1523/ENEURO.0263-16.2017

Copyright (๑) 2017 Martínez et al.

This is an open-access article distributed under the terms of the Creative Commons Attribution 4.0 International license, which permits unrestricted use, distribution and reproduction in any medium provided that the original work is properly attributed.
This study takes advantage of recent developments in transgenic techniques that specifically label GABAergic interneurons to systematically characterize the superficial MEC interneuron population both electrophysiologically and anatomically. Acute brain slices were harvested from glutamic acid decarboxylase 2 (GAD2) + and PV + labeled transgenic mice and whole cell patch clamp techniques were used to measure a variety of electrophysiological features. Post hoc anatomic reconstruction was then conducted using fluorescence staining and two-photon imaging to couple each interneuron's electrophysiological profile with its MEC localization and axonal tree distribution. We find that superficial MEC interneurons can be grouped into four separate groups that have distinct anatomic and electrophysiological profiles. These categories include deep-projecting layer 2/3 slow-firing interneurons, layer $2 / 3$ projecting fast-spiking interneurons, layer 1/2-projecting interneurons and layer 1-projecting superficial interneurons. Our results complement recently published data on the relationship between electrophysiological properties and molecular markers (Ferrante et al., 2017). Together, these two papers represent a large step toward a complete characterization of medial entorhinal interneurons.

\section{Materials and Methods}

\section{Electrophysiology}

All electrophysiology experiments were conducted according to protocols approved by the Institutional Animal Care and Use Committees of the university. Brain slices were harvested from 18-35 d old transgenic mice of either sex. Because no effects of age were evident in our dataset, data were not classified by age of the animal. Two transgenic strains were used: cre-dependent GAD2-IRES-tdTomato transgenic mice (Taniguchi et al., 2011; The Jackson Laboratory, strain 010802), which labeled GAD2-expressing cells and thus facilitated targeting of GABAergic cortical interneurons; and PV-tdTomato transgenic mice (Hippenmeyer et al., 2005; The Jackson Laboratory, strain 008069), which labeled all PVexpressing cells and thus facilitated targeting of the specific PV + genotype in inhibitory interneurons. These mice were anesthetized with isoflurane and decapitated. The brain was then harvested, chilled in sucrosesubstituted artificial CSF (ACSF; 185 sucrose, $2.5 \mathrm{KCl}$, $1.25 \mathrm{NaH}_{2} \mathrm{PO}_{4}, 10 \mathrm{MgCl}_{2}, 25 \mathrm{NaHCO}_{3}, 12.5$ Glucose, 0.5 $\mathrm{CaCl}_{2}$ ), and cut parasagittally into $300-\mu \mathrm{m}$-thick slices using a vibrating microtome (Vibratome VT1200; Leica). Slices were incubated for $15 \mathrm{~min}$ in ACSF $(125 \mathrm{mM} \mathrm{NaCl}$, $2.5 \mathrm{mM} \mathrm{KCl}, 1.25 \mathrm{mM} \mathrm{NaH}_{2} \mathrm{PO}_{4}, 10 \mathrm{mM} \mathrm{MgCl}_{2}, 25 \mathrm{mM}$ $\mathrm{NaHCO}_{3}, 25 \mathrm{mM}$ glucose, and $2 \mathrm{mM} \mathrm{CaCl}_{2}$ ) at $37^{\circ} \mathrm{C}$, and then allowed to recover for at least $30 \mathrm{~min}$ at room temperature. For recordings, slices were transferred to a heated $\left(32-34^{\circ} \mathrm{C}\right)$ slice chamber (Warner Instruments) that is mounted on an upright microscope stage (Olympus BX53; Olympus) and perfused with $95 \% / 5 \% \quad \mathrm{O}_{2} / \mathrm{CO}_{2}$ ACSF. GAD2+/PV + neurons were visualized using fluorescence and whole-cell patch clamp clamped using patch pipettes (5-6 M 2 ) fabricated from borosilicate glass (1.5 O.D. 1.1 I.D.; Sutter Instruments) and filled with artificial intracellular fluid (120 mM K-gluconate, $5 \mathrm{mM}$ 
$\mathrm{MgCl}_{2}, 0.2 \mathrm{mM}$ EGTA, $10 \mathrm{mM}$ HEPES, $20 \mathrm{mM} \mathrm{KCl}, 7 \mathrm{mM}$ di(tris) phosphocreatine, $4 \mathrm{mM} \mathrm{Na}_{2} \mathrm{ATP}$, and $0.3 \mathrm{mM}$ TrisGTP) loaded with biocytin (1\% by weight) for post hoc reconstruction. Presented data were not corrected for the junction potential, which we measured as $11.6 \mathrm{mV}$. Electrode resistance in the bath was $\sim 5 \mathrm{M} \Omega$. The pipette capacitance was compensated for automatically by the MultiClamp 700B/CV-7 system. The bridge balance was applied to correct for series electrode resistance under whole-cell patch clamp $(\sim 10 \mathrm{M} \Omega)$. Cells were patched for at least $30 \mathrm{~min}$ to ensure complete biocytin fill. Following electrophysiological trials, brain slices were perfused in 4\% paraformaldehyde (PFA) for 16-24 $\mathrm{h}$, then washed in PBS (137 mM NaCl, $2.7 \mathrm{mM} \mathrm{KCl,} 10 \mathrm{mM} \mathrm{Na}_{2} \mathrm{HPO}_{4}$, and $1.8 \mathrm{mM} \mathrm{KH}_{2} \mathrm{PO}_{4}$ ) three times for $15 \mathrm{~min}$ each and stored in $4^{\circ} \mathrm{C}$ for later staining.

\section{Post hoc anatomic reconstruction}

Brain slices were incubated for $3 \mathrm{~h}$ in a PBS solution containing $3 \mu \mathrm{g} / \mathrm{ml}$ streptavidin Alexa Fluor 488 (Invitrogen) and 2\% Triton X-100 (by volume). Slices were then washed in PBS three times for 15 min each and mounted on slides using a Mowiol mounting medium. At least $24 \mathrm{~h}$ after mounting, slides were imaged using a two-photon microscope (Ultima Intravital, Bruker), with excitation wavelength set to $810 \mathrm{~nm}$ and a 520-nm low-pass filter. Alexa Fluor 488-filled cells were localized in the brain slice and a z-stack of $585 \times 585 \mu \mathrm{m}$ raster-scanned images was acquired, covering the entire range of the soma and neuronal processes (usually 100-200 $\mu \mathrm{m}$ ). This z-stack was projected onto a single composite image and endowed with a dark-cell, light-background look-up table to aid axonal visualization (Fig. 1). to describe the anatomic features of each neuron, soma depth was measured and the extent of the axonal tree was described with a rectangular approximation using the z-projected image (Fig. 1).

\section{Electrophysiological protocols and data analysis}

We used established methods to characterize the electrophysiological features of patched cells. All electrophysiological protocols were conducted in current clamp and were performed within 30 min of breaking into the cell to begin the whole cell patch clamp recording.

Input resistance, time constant, sag ratio, and resting membrane potential

A bias current was applied in current clamp to hold the cell to $-70 \mathrm{mV}$. Five 1 -s negative current pulses (with a 2-s rest time) were injected to hyperpolarize the cell to approximately $-80 \mathrm{mV}$ (between -20 and $-50 \mathrm{pA}$, depending on input resistance). The resulting steady-state hyperpolarization from $-70 \mathrm{mV}$ was divided by the applied current to calculate the input resistance. To determine the membrane time constant, a single-order exponential was fit to the membrane response (from start to peak polarization) to the hyperpolarizing current. The sag ratio was determined by dividing the maximum voltage hyperpolarization (the sag) by the steady state hyperpolarization during the pulse. The resting membrane potential was measured for every cell by placing the cell in current clamp, injecting no current, and measuring the membrane potential.

\section{$A P$ half-width, $A P$ rise time, and spike afterhyperpolarizing} potential $(A H P)$

A depolarizing bias current was injected to elicit APs just above firing threshold. The average of $50-100$ total APs recorded in a 30-40 s recording was used to describe the AP shape. First, the AP half-width was determined by determining the AP height from the AP upstroke (defined as the point at which the second derivative of the AP with respect to time was at its maximum) to the peak and calculating the duration between passing the halfheight on the depolarizing phase and passing the halfheight on the hyperpolarizing phase is the AP half-width. The AP rise time was calculated as the time required for the AP to go from $20 \%$ of its total height to $80 \%$ of its total height. The AHP was measured as the membrane potential difference between the AP upstroke initiation and the most hyperpolarized membrane potential immediately following the AP.

\section{Firing threshold}

The cell was hyperpolarized to $-80 \mathrm{mV}$ and a $50 \mathrm{pA} / \mathrm{s}$ current ramp for 10-20 s was applied, depending on the input resistance. The firing threshold was determined as the membrane potential at the upstroke of the first AP in the ramp.

\section{Impedance}

The cell was hyperpolarized to $-80 \mathrm{mV}$ and a $15 \mathrm{~s}$ filtered white noise current trace (filtered at $200 \mathrm{~Hz}$ and set to $50-100 \mathrm{pA}$ in amplitude to elicit a membrane response $\sim 5 \mathrm{mV}$ in amplitude) was injected. We used a low pass filtered white noise, as described in Fernandez et al. (2015), where the current signal was constructed in the frequency domain using a frequency amplitude equal to $1 /(1+(f / 200))$, where $f$ is the frequency. The voltage trace and the resulting injected current trace were each converted into the frequency domain (using a fast Fourier transform). The frequency domain of the voltage was divided by the frequency domain of injected current trace and magnitude component of the resulting trace was used as the impedance. This procedure was repeated several times at increasing depolarized membrane potentials until the cell was near its firing threshold. The impedance change was measured by calculating the average impedance between 1 and $10 \mathrm{~Hz}$ for the most depolarized trace (labeled "perithreshold") and dividing it by the average impedance for the $-80 \mathrm{mV}$ trace in the same frequency band.

Frequency-current (F-I) gain, peak firing rate, minimum firing rate, adaptation ratio, and rheobase

A bias current was applied in current clamp to polarize the cell to $-70 \mathrm{mV}$. A series of one-second current pulses (with a four second rest between pulses) was injected to determine the F-I relationship of the cell. These current pulses ranged from -100 to up to $1500 \mathrm{pA}$, depending on what current amplitude was required to reach a firing rate plateau, and were introduced in $20 \mathrm{pA}$ increments. The peak firing rate was the fastest firing frequency recorded during the F-I trial. The minimum firing rate was measured 


\section{Electrophysiological description}
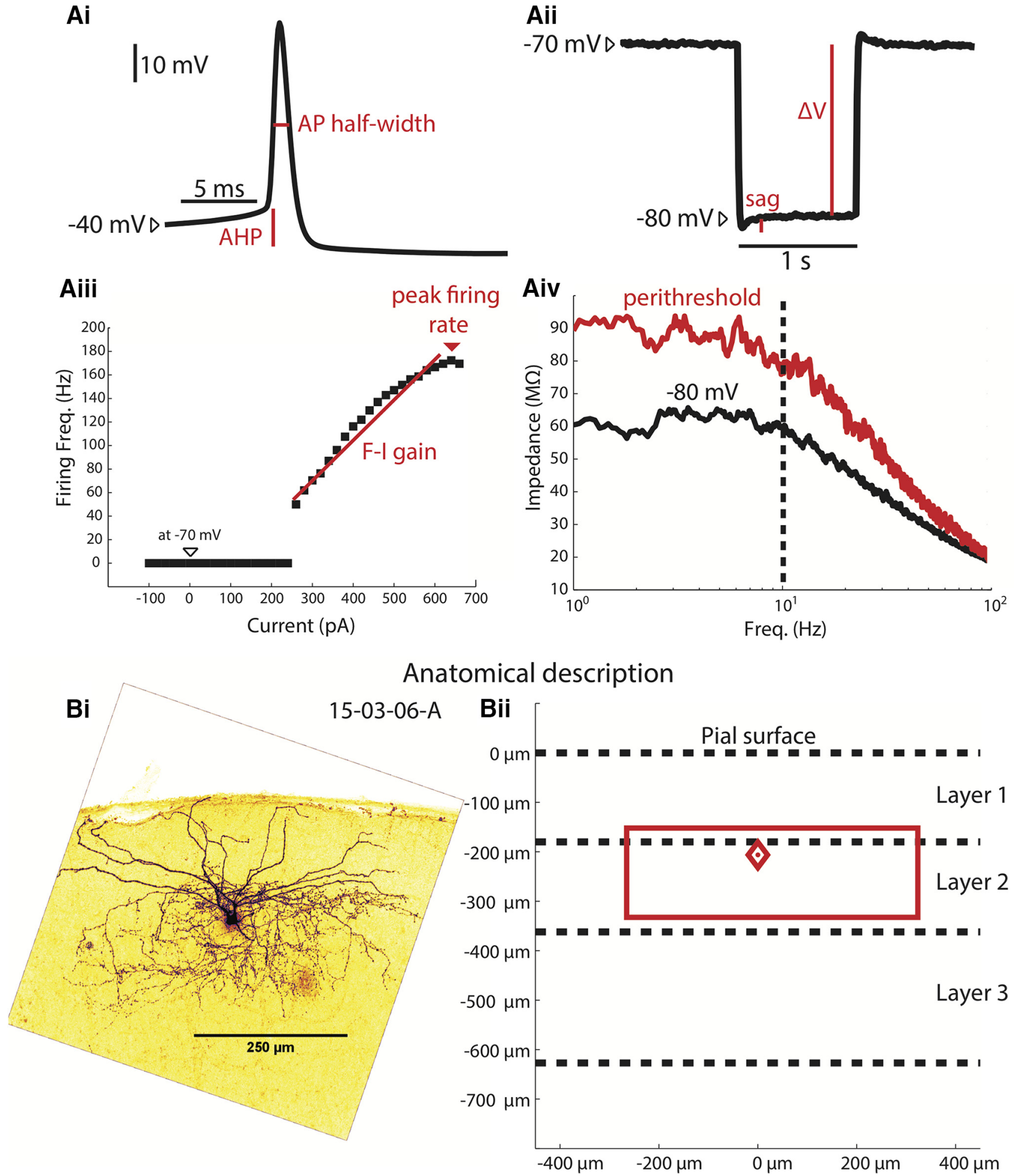

Figure 1. MEC interneuron electrophysiological and anatomic characterization. Ai, Depolarizing current was injected to elicit firing and resulting APs were characterized. AP half-widths are measured at half the height of the AP (using the AP initiation upstroke, or "knee," as the base). The duration between passing the half-height on the depolarizing phase and passing the half-height on the hyperpolarizing phase is the AP half-width. The AHP is measured as the membrane potential difference between the AP knee and the most hyperpolarized membrane potential immediately following the AP. Aii, One-second-long hyperpolarizing pulses are injected to hyperpolarize the cell from $-70 \mathrm{mV}$ to approximately $-80 \mathrm{mV}$. The resulting voltage deflection $\Delta \mathrm{V}$ is divided by the injected current $\Delta \mathrm{l}$ to calculate the input resistance. The sag ratio was defined as $\Delta \mathrm{V} /(\mathrm{sag}+\Delta \mathrm{V})$. Aiii, The F-I relationship was described injecting 


\section{continued}

progressively increasing current pulses and measuring the resulting firing rate. The slope between the first non-zero F-I trial and the peak firing trial is the F-I gain. The fastest firing rate elicited by the current pulses is the peak firing rate. Aiv, The impedance spectra are measured at both $-80 \mathrm{mV}$ and near threshold are used to calculate the impedance change. The impedance between 1 and 10 $\mathrm{Hz}$ of the perithreshold spectrum is divided by the same impedance band of the rest spectrum. The dashed line indicates the upper band for the impedance change calculation, $10 \mathrm{~Hz}$. Bi, A z-stack projection of a biocytin filled, Alexa Fluor 488-labeled MEC interneuron is used to determine the location of the soma and estimate the extent of the axonal tree. Bii, The neuron in Bi is described using a diamond to indicate the depth of the soma (relative to the pial surface) and a rectangle to describe the laminar and columnar extent of the neuron's axonal projection, both in red. Dashed line indicate the average depth of layers 1-3. This neuron has a soma at the layer $1 / 2$ border ( $\sim 200 \mu \mathrm{m}$ deep), and its axonal tree extends from $\sim 170$ to $350 \mu \mathrm{m}$ in depth and is $\sim 500 \mu \mathrm{m}$ wide.

as the lowest non-zero firing rate recorded during the trial. This measurement illustrates any large discontinuities in spike frequency associated with the spike threshold transisiton. To determine the F-I gain, we determined both the F-I trial point at which the minimum firing rate was achieved and the F-I trial point at which the firing rate asymptote began. A linear regression fit for all the F-I trials between these two points (inclusively) was calculated with the least-squares "polyfit" function in MATLAB for a first order polynomial. The slope of this fit was taken to be the F-I gain. The adaptation ratio was measured as the ratio of the first three interspike intervals to the last three interspike intervals in the median firing F-I step. For example, if an F-I trial had 11 steps in which the interneuron fired at least one spike, then the 6th trial would be used to measure the adaptation ratio. The rheobase was estimated using the F-I trial by detecting the first current pulse to elicit an AP. The magnitude of that current pulse was taken to be the rheobase for that cell.

\section{Grouping methodology \\ Principal component analysis (PCA)}

PCA (Jolliffe, 2002) was used to prevent correlations between the different measured values in the electrophysiological/anatomic dataset. The electrophysiological features used for this analysis were: input resistance, peak firing rate, rising time constant, change in impedance and F-I gain. These electrophysiological measurements were selected as they represent both the passive and active membrane properties of the cell populations, while not including several measurements that could be correlated. The anatomic features used were: soma depth, the most superficial extent of the axonal tree, the deepest extent of the axonal tree, and the axonal width. Because including rheobase and resting membrane potential had minimal effect on the outcome of k-means clustering described below (changing the classification of only $4 / 106$ cells), we did not include these variables. All these features were z-scored (i.e. mean-subtracted and divided by the standard deviation) before the analysis. Each cell was treated as an observation with each feature a variable. The "princomp" function in MATLAB was used to calculate the transformation. We used four principal components to account for $80 \%$ of the variance. Increasing this number did not change the k-means classification outcome. Using three, two, or one principal component changed the k-means outcome for 3,39 , and 45 of 106 classified cells. Thus, we conclude that using three to four components is optimal for this dataset, and used four to meet the standard criterion of accounting for $80 \%$ of variance.

\section{K-means clustering analysis}

K-means clustering analysis (MacQueen, 1967) was used on the first four principal components of the above dataset to group cells. The "kmeans" function in MATLAB 2016a was used with squared Euclidean distance as the metric, the kmeans ++ seeding algorithm (Arthur and Vassilvitskii, 2007), and 100 iterations for each operation to ensure convergence. Silhouette scores were calculated using the "silhouette" function in MATLAB. The silhouette score is a measure of similarity of a point to points within its own cluster and of dissimilarity of a point to points outside of its own cluster (Rousseeuw, 1987). For a given cell $i$, it is calculated as $\mathrm{s}(i)=(\mathrm{b}(i)-\mathrm{a}(i)) /$ maximum $[\mathrm{a}(i), \mathrm{b}(i)]$, where $\mathrm{a}(i)$ is the average distance between cell $i$ and all other cells in its cluster and $b(i)$ is the shortest distance between cell $i$ and any cell not in $i$ 's cluster. The range of values ranges from -1 to 1 . A higher score (closer to 1 ) indicates high similarity within cluster and dissimilarity outside of cluster, whereas a lower score (closer to -1) indicates low similarity within cluster and dissimilarity outside of cluster (suggesting the data point was misclassified).

\section{Group comparisons}

When comparing electrophysiological and anatomic features among different cell groups, reported $p$ values were calculated using a the nonparametric Kruskal-Wallis one-way ANOVA test. The $F$ value and $p$ values for all comparisons are shown in Table 1.

\section{Alternative methods of clustering}

In addition to the combined data k-means clustering method used in this study, we explored several different approaches to the clustering problem. First, we used hierarchical clustering to group the interneuron population and compared the results to those arrived at using $\mathrm{k}$-means clustering. The first four principal components of the combined anatomic/electrophysiological dataset (five electrophysiological measures and four anatomic measures) were clustered using the unweighted pair group method with arithmetic mean (UPGWA) for hierarchical clustering (Sokal, 1958), using the least squared Euclidean distance to separate interneurons. As this method sequentially separates the population into different hierarchies, it is possible from one analysis to group interneurons into a few large clusters or several smaller clusters. For the purposes of comparing our results to those of k-means clustering, the cutoff for differentiating clusters was set to $60 \%$ of the maximum distance between any two interneurons. That is, all interneurons within a single 
Table 1. Statistical tests

\begin{tabular}{lll}
\hline Characteristic & $F$ value & $p$ value \\
Input resistance $(\mathrm{M} \Omega)$ & 72.93 & $1.0 \mathrm{E}-15$ \\
Time constant, falling $(\mathrm{ms})$ & 75.86 & $2.4 \mathrm{E}-16$ \\
Time constant, rising $(\mathrm{ms})$ & 76.05 & $2.2 \mathrm{E}-16$ \\
Rebound amplitude $(\mathrm{mV})$ & 25.08 & $1.5 \mathrm{E}-05$ \\
Firing threshold $(\mathrm{mV})$ & 12.26 & $6.5 \mathrm{E}-03$ \\
Resting membrane potential $(\mathrm{mV})$ & 37.26 & $4.1 \mathrm{E}-08$ \\
A.P. rise time $(\mathrm{ms})$ & 57.61 & $1.9 \mathrm{E}-12$ \\
A.P. half-width $(\mathrm{ms})$ & 69.29 & $6.1 \mathrm{E}-15$ \\
Spike AHP $(\mathrm{mV})$ & 16.44 & $9.2 \mathrm{E}-04$ \\
F-I gain $(\mathrm{Hz} / \mathrm{nA})$ & 42.62 & $3.0 \mathrm{E}-09$ \\
Peak firing rate $(\mathrm{Hz})$ & 74.60 & $4.4 \mathrm{E}-16$ \\
Lowest firing rate $(\mathrm{Hz})$ & 54.33 & $9.6 \mathrm{E}-12$ \\
Rheobase $(\mathrm{pA})$ & 59.90 & $6.2 \mathrm{E}-13$ \\
Adaptation ratio & 22.01 & $6.5 \mathrm{E}-05$ \\
Change in impedance $(\%)$ & 14.42 & $2.4 \mathrm{E}-03$ \\
Sag ratio & 33.51 & $2.5 \mathrm{E}-07$ \\
Soma depth $(\mu \mathrm{m})$ & 42.38 & $3.3 \mathrm{E}-09$ \\
Axonal tree, superficial $(\mu \mathrm{m})$ & 29.87 & $1.5 \mathrm{E}-06$ \\
Axonal tree, deep $(\mu \mathrm{m})$ & 29.77 & $1.5 \mathrm{E}-06$ \\
Axonal tree, width $(\mu \mathrm{m})$ & 25.09 & $1.5 \mathrm{E}-05$ \\
\end{tabular}

All characteristics were tested for normality using the Kolmogorov-Smirnov test, and none were found to be normally distributed. The nonparametric Kruskal--Wallis test was used to compare groups, and the $F$ value and $p$ value for each test is shown.

cluster have to be no further than $60 \%$ of the maximum distance measured in this population; interneurons with a greater distance must be in separate clusters.

Second, we explored clustering the interneurons using either only anatomic data or electrophysiological data, as opposed to combining both data types into one analysis. We separated out the four anatomic and five electrophysiological measures and conducted separate analyses. For both sets of data, we z-scored the measurements and conducted PCA, as described previously. For k-means clustering analysis, we set the cluster number to four, to match with the optimal cluster number for the combined analysis. The optimal cluster number was determined by varying the set number of clusters and measuring the mean silhouette score of all the clusters. The number of clusters that yielded that highest mean silhouette score was set to be the optimal cluster number.

To compare different distributions, it was necessary to determine the optimal correspondence between clusters of one distribution to that of the combined k-means clustering distribution used in the study. We established the optimal correspondence by testing every possible permutation for assignment overlap (percentage of interneurons assigned to the same cluster in both distributions), and choosing the permutation of highest overlap.

\section{Validation of transgenic mouse models}

In order to validate the transgenic mice line, immunohistochemistical analysis was performed. Mice were injected with an overdose of pentobarbital sodium/phenytoin sodium (Euthasol, Vibrac Animal Health) and transcardially perfused with $0.05 \mathrm{M}$ PBS followed by $4 \%$ PFA in $0.05 \mathrm{M}$ PBS. Brains were removed and post fixed in the PFA for $4 \mathrm{~h}$. Brains were transferred to $30 \%$ sucrose solution in $0.05 \mathrm{M} \mathrm{KPBS}$ solution and incubated over night to be cryoprotected. Each brain was flash froze in OCT and sliced into $40-\mu \mathrm{m}$ horizontal slices. Slices from GAD2-TdTomato and PV-Tdtomato animals were, respectively, stained with markers for GAD2 (Invitrogen, PA5-22260) and PV (Swant, PV25) followed by Alexa Fluor 488 goat-antirabbit secondary antibody. Imaging was performed using two-photon imaging system (Thorlabs) with a mode-locked Ti:Sapphire laser (Chameleon Ultra II; Coherent) set to wavelengths between 900 and $925 \mathrm{~nm}$ using 20× water immersion NA 1.0 (Olympus) objective lens. For the PV slices, z-stacks were taken by imaging at $0.5-\mu \mathrm{m}$ intervals through the regions interest of the slice. For the GAD2 slices, image was taken at a single plane. Cells were counted by analyzing two nonoverlapping regions of MEC on each slice for each animal.

\section{Results}

\section{Interneuron characterization}

Interneurons of the mouse superficial MEC were systematically patched, electrophysiologically characterized, and then anatomically reconstructed to better understand the local inhibitory components of this brain region. Because interneurons make up a small $(<10 \%)$ portion of all the MEC (Gatome et al., 2010), transgenic mice labeling GAD2 + and PV + cells were used to target the neuron subpopulation. For each interneuron, passive properties (like input resistance at rest, time constant, and sag ratio) and active properties (like AP shape and F-I relationships) were measured in the current clamp configuration (Fig. $1 A)$, and the neuron was stained post hoc with an Alexa Fluor 488 fluorescent marker. The neuron was then reconstructed in a three-dimensional z-stack using a two-photon microscope (Fig. 1B). In all, each neuron had sixteen electrophysiological features and four anatomic features recorded (Table 2).

The in vitro immunohistochemical validation of the transgenic lines showed that the vast majority $(91 \%)$ of GAD2-tdTomato-positive cells were labeled by GAD2 markers. Similarly, nearly all (97\%) PV-tdTomato-positive cells were labeled by PV markers. Both results can be seen in Figure 2. The study yielded a total of 106 interneurons with complete electrophysiological and anatomic profiles. For the 106 cell population, the distribution of each measured characteristic is shown in Figure 3. Of these, 80 cells were acquired using GAD2 + mice and 26 were acquired in PV+ mice. Cells that had incomplete or inadequate electrophysiological trials were not analyzed. Common causes of incomplete electrophysiological characterization included cell death during experiment, incomplete pipette to cell seal, and noise artifacts that corrupted the data acquisition. Cells that exhibited large changes in resting membrane potential at any point during the trials were discarded. Cells that had incomplete anatomic reconstructions, particularly those where the axonal tree was not visible, were also discarded. Common issues with anatomic reconstruction included incomplete anatomic fills and inadequate staining. 
Table 2. Electrophysiological and anatomic characteristics for all four interneuron clusters with statistical comparisons

\begin{tabular}{|c|c|c|c|c|c|c|c|c|c|c|}
\hline Cluster & 1 & 2 & 3 & 4 & 1,2 & 1,3 & 1,4 & 2,3 & 2,4 & 3,4 \\
\hline$n$ & 30 & 29 & 16 & 31 & & & & & & \\
\hline \multirow[t]{2}{*}{ Input resistance $(\mathrm{M} \Omega)$} & 220.2 & 85.7 & 274.4 & 155.1 & & & & & & \\
\hline & 12.3 & 5.1 & 14.9 & 6.2 & $p<0.01$ & n.s. & $p<0.05$ & $p<0.01$ & $p<0.01$ & $p<0.01$ \\
\hline \multirow[t]{2}{*}{ Time constant, falling (ms) } & 13.7 & 5.1 & 15.8 & 8.9 & & & & & & \\
\hline & 0.8 & 0.2 & 0.9 & 0.4 & $p<0.01$ & n.s. & $p<0.01$ & $p<0.01$ & $p<0.01$ & $p<0.01$ \\
\hline \multirow[t]{2}{*}{ Time constant, rising (ms) } & 14.1 & 5.2 & 16.2 & 9.5 & & & & & & \\
\hline & 0.8 & 0.2 & 1.0 & 0.4 & $p<0.01$ & n.s. & $p<0.01$ & $p<0.01$ & $p<0.01$ & $p<0.01$ \\
\hline \multirow[t]{2}{*}{ Rebound amplitude (mV) } & 0.75 & 0.41 & 1.48 & 0.62 & & & & & & \\
\hline & 0.08 & 0.03 & 0.20 & 0.10 & $p<0.05$ & n.s. & n.s. & $p<0.01$ & n.s. & $p<0.01$ \\
\hline \multirow[t]{2}{*}{ Firing threshold (mV) } & -38.7 & -36.7 & -40.8 & -35.6 & & & & & & \\
\hline & 0.9 & 1.0 & 1.1 & 1.0 & n.s. & n.s. & n.s. & $p<0.05$ & n.s. & $p<0.05$ \\
\hline \multirow[t]{2}{*}{ Resting membrane potential (mV) } & -66.3 & -71.4 & -55.5 & -66.8 & & & & & & \\
\hline & 1.0 & 1.0 & 2.1 & 1.1 & $p<0.01$ & $p<0.01$ & n.s. & $p<0.01$ & $p<0.05$ & $p<0.01$ \\
\hline \multirow[t]{2}{*}{ A.P. rise time (ms) } & 0.273 & 0.192 & 0.253 & 0.292 & & & & & & \\
\hline & 0.006 & 0.003 & 0.013 & 0.009 & $p<0.01$ & n.s. & n.s. & $p<0.01$ & $p<0.01$ & n.s. \\
\hline \multirow[t]{2}{*}{ A.P. half-width (ms) } & 1.085 & 0.526 & 0.832 & 1.151 & & & & & & \\
\hline & 0.038 & 0.010 & 0.067 & 0.047 & $p<0.01$ & n.s. & n.s. & $p<0.01$ & $p<0.01$ & $p<0.05$ \\
\hline \multirow[t]{2}{*}{ Spike AHP (mV) } & 16.2 & 19.9 & 19.3 & 20.8 & & & & & & \\
\hline & 1.0 & 0.5 & 1.0 & 0.7 & $p<0.05$ & n.s. & $p<0.01$ & n.s. & n.s. & n.s. \\
\hline \multirow[t]{2}{*}{$\mathrm{F}-\mathrm{I}$ gain $(\mathrm{Hz} / \mathrm{nA})$} & 153.0 & 281.9 & 373.9 & 162.0 & & & & & & \\
\hline & 18.9 & 15.8 & 51.4 & 13.0 & $p<0.01$ & $p<0.01$ & n.s. & n.s. & $p<0.01$ & $p<0.01$ \\
\hline \multirow[t]{2}{*}{ Peak firing rate $(\mathrm{Hz})$} & 57.4 & 279.2 & 111.5 & 75.3 & & & & & & \\
\hline & 5.1 & 8.6 & 8.4 & 5.6 & $p<0.01$ & $p<0.01$ & n.s. & $p<0.01$ & $p<0.01$ & n.s. \\
\hline \multirow[t]{2}{*}{ Lowest firing rate $(\mathrm{Hz})$} & 10.6 & 79.2 & 13.7 & 13.9 & & & & & & \\
\hline & 2.3 & 6.0 & 3.0 & 2.3 & $p<0.01$ & n.s. & n.s. & $p<0.01$ & $p<0.01$ & n.s. \\
\hline \multirow[t]{2}{*}{ Rheobase (pA) } & 185.7 & 380.7 & 118.8 & 262.6 & & & & & & \\
\hline & 15.7 & 22.2 & 12.3 & 13.2 & $p<0.01$ & n.s. & $p<0.05$ & $p<0.01$ & $p<0.05$ & $p<0.01$ \\
\hline \multirow[t]{2}{*}{ Adaptation ratio } & 0.783 & 0.878 & 1.335 & 0.917 & & & & & & \\
\hline & 0.153 & 0.007 & 0.730 & 0.153 & $p<0.01$ & n.s. & n.s. & $p<0.01$ & $p<0.05$ & n.s. \\
\hline \multirow[t]{2}{*}{ Sag ratio } & 0.936 & 0.945 & 0.888 & 0.936 & & & & & & \\
\hline & 0.006 & 0.004 & 0.013 & 0.008 & n.s. & $p<0.05$ & n.s. & $p<0.01$ & n.s. & $p<0.01$ \\
\hline \multirow[t]{2}{*}{ Change in impedance (\%) } & 64.8 & 133.1 & 136.5 & 51.6 & & & & & & \\
\hline & 10.6 & 12.7 & 15.7 & 9.2 & $p<0.01$ & $p<0.01$ & n.s. & n.s. & $p<0.01$ & $p<0.01$ \\
\hline \multirow[t]{2}{*}{ Soma depth $(\mu \mathrm{m})$} & 327.0 & 304.9 & 274.6 & 191.6 & & & & & & \\
\hline & 14.2 & 16.1 & 19.7 & 8.9 & n.s. & n.s. & $p<0.01$ & n.s. & $p<0.01$ & $p<0.05$ \\
\hline \multirow[t]{2}{*}{ Axonal tree, superficial $(\mu \mathrm{m})$} & 296.4 & 168.4 & 107.0 & 114.0 & & & & & & \\
\hline & 21.5 & 19.8 & 33.2 & 25.2 & $p<0.01$ & $p<0.01$ & $p<0.01$ & n.s. & n.s. & n.s. \\
\hline \multirow[t]{2}{*}{ Axonal tree, deep $(\mu \mathrm{m})$} & 446.4 & 298.0 & 318.0 & 252.9 & & & & & & \\
\hline & 23.0 & 16.4 & 35.6 & 25.7 & $p<0.01$ & $p<0.05$ & $p<0.01$ & n.s. & n.s. & n.s. \\
\hline \multirow[t]{2}{*}{ Axonal tree, width $(\mu \mathrm{m})$} & 361.4 & 513.0 & 440.6 & 472.2 & & & & & & \\
\hline & 21.7 & 12.8 & 30.8 & 20.1 & $p<0.01$ & n.s. & $p<0.01$ & n.s. & n.s. & n.s. \\
\hline
\end{tabular}

The electrophysiological and anatomic characteristics of all four clusters are shown, along with the associated $p$ values from a Kruskal--Wallis test (as described in Materials and Methods). Each row shows the average value for a different electrophysiological or anatomic measurement. The standard error associated with that measurement is located below the average value. Each column for the left half of the table shows the measurements for each of the four clusters. On the right half of the table, the $p$ value for the Kruskal--Wallis test is shown for matched pairs. For example, column "1,2" shows the $p$ values for the test between clusters 1 and 2 for each characteristics; $p<0.05$ are highlighted in blue. Tests that showed no significance are shown as n.s.

\section{Classifying interneurons into distinct groups K-means clustering}

Given that the large dataset included 106 interneurons each with 16 electrophysiological features and four anatomic features, PCA was used to reduce the variation of the data into fewer dimensions. PCA was conducted using five selected electrophysiological and four anatomic measurements (see Materials and Methods). Since this diverse set of measurements vary greatly in mean and variance, all measurements were $z$-scored to standardize the PCA variables to a mean of 0 and variance of 1 . To reduce the dimensionality of the dataset, subsequent analyses used only the top four ranked principal compo- nents, which altogether accounted for $80 \%$ of the variability in the data. The first four principal components accounted for $29.4 \%, 24.7 \%, 16.7 \%$, and $9.2 \%$ of the variability, respectively. The next five principal components accounted for $6.7 \%, 4.7 \%, 3.7 \%, 2.7 \%$, and $1.9 \%$ of variability, respectively. The relationships among the four principal components have been plotted in Figure $4 A$.

The resulting principal components were then used to group cells into distinct clusters. K-means clustering analysis was performed as described previously. Given that $\mathrm{k}$-means clustering requires the number of clusters as an input, it was necessary to first determine the optimal number of clusters in which to divide the dataset. K- 


\section{A}
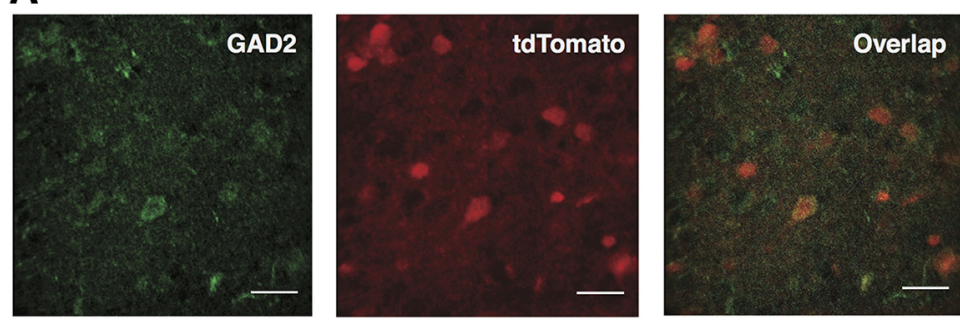

B
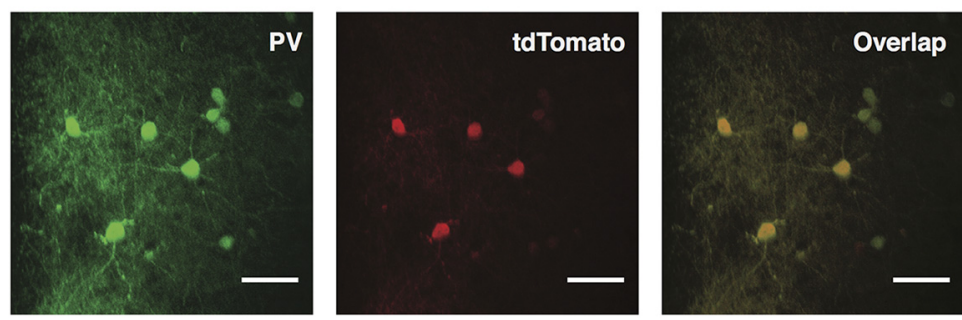

Figure 2. Immunohistochemical validation of transgenic mouse lines. $\boldsymbol{A}$, Representative image showing overlap of GAD2 labeling with tdTomato cells of transgenic mice. The great majority of the tdTomato-positive cells are labeled for GAD2 as well ( $91.2 \%$ of 74 cells counted; $n=2$ animals). Scale bar: $25 \mu \mathrm{m}$. B, Representative image showing overlap of staining for PV with tdTomato cells. Nearly all tdTomato-positive cells are labeled for PV as well ( $97 \%$ of 74 cells counted; $n=2$ animals). Scale bar: $15 \mu \mathrm{m}$.

means clustering was thus conducted on a range of cluster number inputs, from only two clusters to up to 16 clusters. For each cluster number input, a silhouette score was calculated for all cells. The silhouette score is a measure of the cluster "fit": it is high when a data point (in this case a cell) is more similar to data points within its cluster than those outside of its cluster. The average silhouette score for all 106 interneurons in each of the 2-16 cluster k-means analyses was calculated to validate the cluster fit (Fig. 4Bi). The highest mean silhouette score was achieved when four clusters were assigned to the dataset, suggesting that the analysis is optimal with four clusters.

The resultant silhouette scores are shown in Figure 4Bii. The GAD2-PV cell distribution for these clusters is shown in Figure 4Biii. Cluster 1 has a total of 30 cells, cluster 2 has 29 cells, cluster 3 has 16 cells, and cluster 4 has 31 cells. Notably, the k-means clustering analysis placed all $26 \mathrm{PV}+$ cells in the dataset into cluster 2 . The fact that all $\mathrm{PV}+$ cells were placed in a single cluster and that the cluster itself was almost entirely (26 out of $29,90 \%$ ) comprised of verified PV + cells lends further support to the PCA/k-mean clustering method used in this study. This result also suggests that PV + cells are a very homogeneous group, representing a relatively small fraction of the GABAergic cells, and anatomically and electrophysiologically distinct from the others.

\section{Hierarchical clustering}

We conducted UPGWA hierarchical clustering on the same four-dimensional principal component anatomy/ electrophysiology data used for k-means clustering in the study. Hierarchical clustering separated the 106 interneuron population into the dendrogram in Figure $5 \mathrm{Ai}$, with each end point representing a single interneuron and the branch connections indicating linkages between interneu- rons. This created various levels ("hierarchies") into which the population could be grouped. Any separation would be based on the minimum required linkage between interneurons for these interneurons to be grouped into the same cluster. By visual inspection, we tested a range of cutoff distances to yield four clusters of similar sample sizes to the k-means clusters used in the study. We therefore set the cutoff at $60 \%$ of the maximum distance between any two interneurons in the population. The resulting seven clusters are colored differently in Figure $5 \mathrm{Ai}$. to adequately compare these clusters with those in the k-means clustering analysis, we inspected all possible permutations $(7 !=5040)$ for maximum overlap. This produced the corresponding k-means cluster labels for the hierarchical clusters shown in Figure $5 \mathrm{Ai}$.

The hierarchical clustering analysis had $82 \%$ overlap with the k-means clustering analysis, meaning $82 \%$ of interneurons were placed in the same cluster in both analyses. This result indicates substantial agreement in the results between both methods. The distributions for each clustering analysis are shown in Figure 5Aii. Cluster 1 had 26 interneurons in hierarchical clustering, 91\% of which were categorized into the k-means cluster 1; in turn, there were 30 interneurons in k-means cluster 1, $70 \%$ of which were classified into hierarchical cluster 1. The interneurons that were not classified into the same cluster are displayed as black lines leading to the corresponding cluster in Figure 5Aii. Both cluster 2 populations contained all $\mathrm{PV}+$ cells, as shown by the red whiskers indicating PV + cells in Figure 5Aii. Hierarchical cluster 2 had 31 interneurons, 97\% classified into k-means cluster 2; in turn, k-means cluster 2 had 29 interneurons, all of which were classified into hierarchical cluster 2. This shows a high degree of agreement in the predominantly $\mathrm{PV}+$ cluster 2 . Cluster 3 in hierarchical clustering had 6 

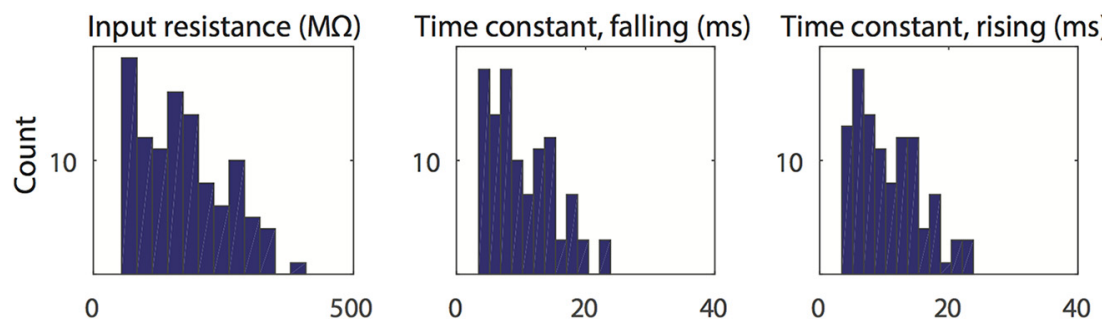

Rebound amplitude $(\mathrm{mV})$

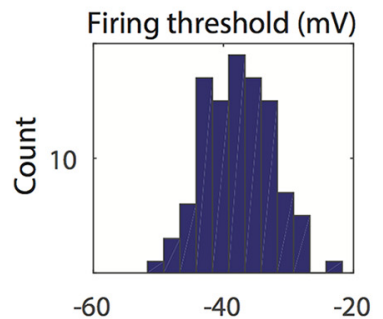

Resting mem. potential (mV)
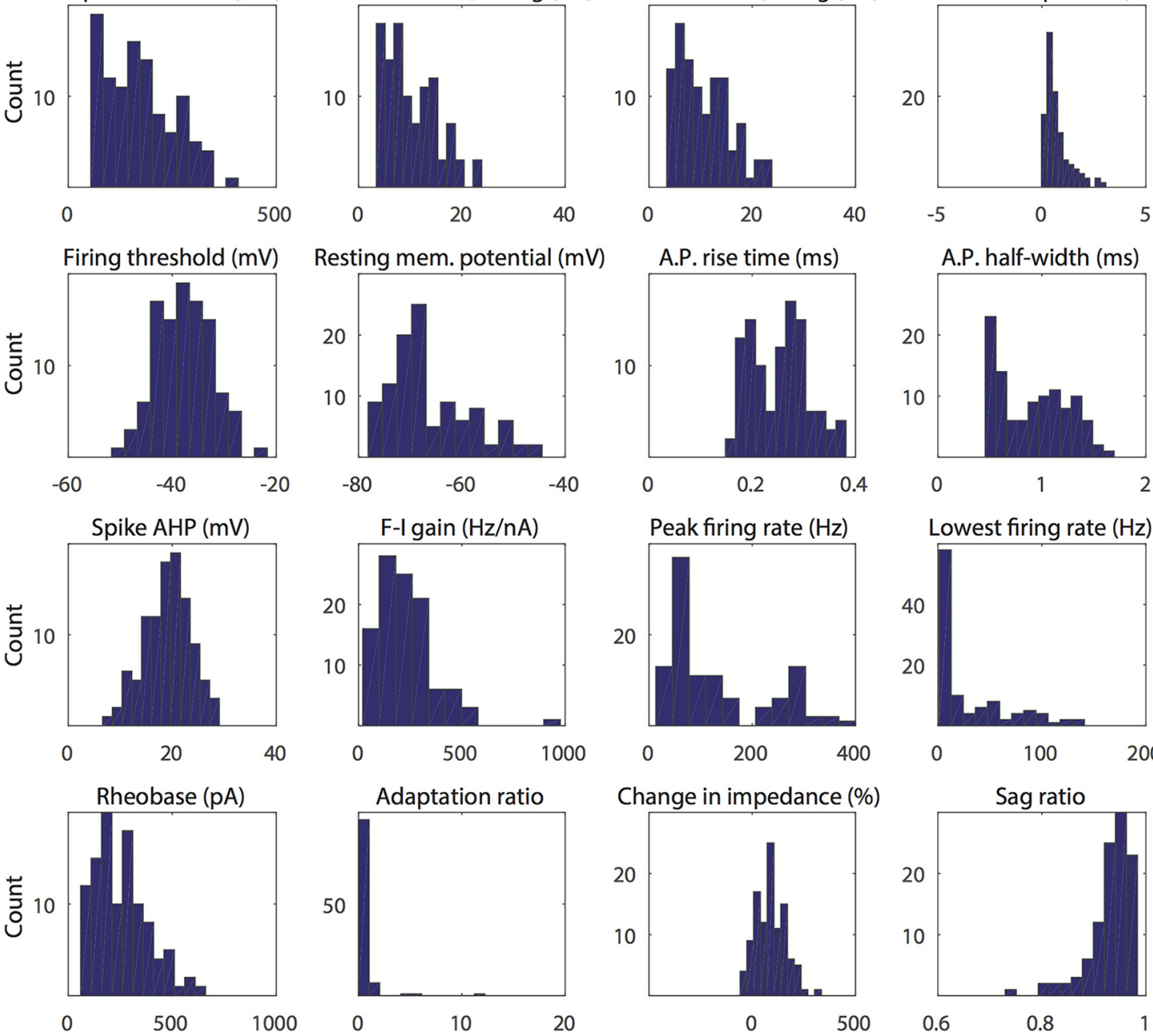

Change in impedance (\%)
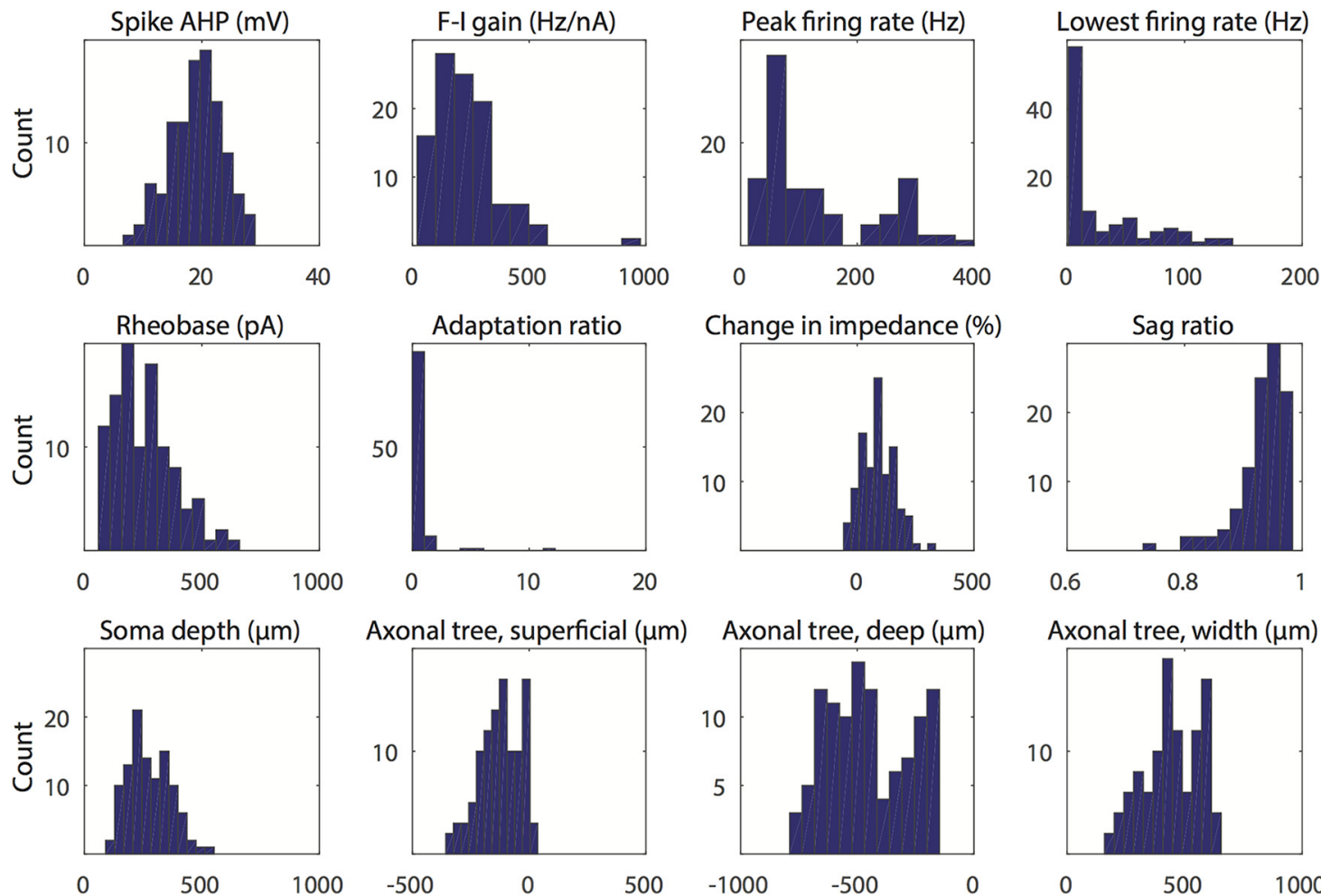

Axonal tree, superficial $(\mu \mathrm{m})$

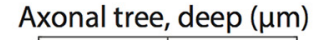

Axonal tree, width $(\mu \mathrm{m})$
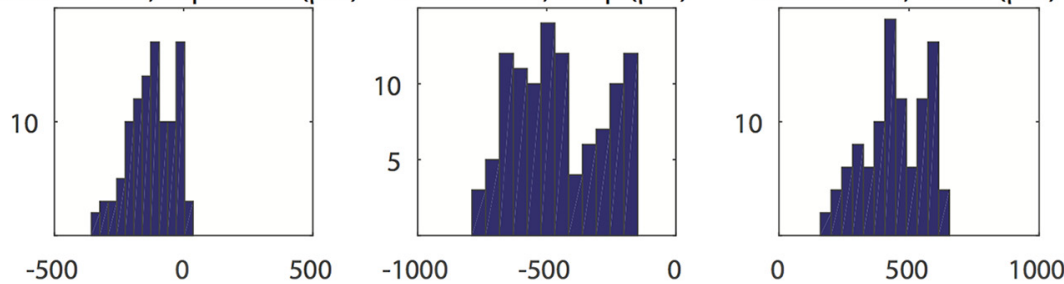

Figure 3. Histogram distributions for electrophysiological and anatomic characteristics. The distributions of the 20 electrophysiological and anatomic characteristics for all 106 superficial MEC interneurons are displayed in histogram form, with each characteristic binned into 12 groups.

interneurons, all of which were classified in cluster 3 of the k-means analysis. The k-means cluster 3 had 16 interneurons, only $38 \%$ of which were classified into cluster 3 in hierarchical clustering (the theoretical maximum given the different group sizes). Hierarchical cluster 4 had 37 interneurons, $82 \%$ of which were classified into k-means cluster 4 . This cluster had 31 interneurons all of which were classified into hierarchical cluster 4 .

\section{Separate k-means clustering of anatomic and electrophysiological data}

We conducted separate analyses using only either anatomic or electrophysiological data using the same PCA and k-means clustering analysis as the study. We matched the resulting four clusters from each analysis to the combined, four-cluster k-means analysis.

First, we compared the clustering results using either only the anatomy or electrophysiology data (Fig. 1Bi). These distributions showed only a $58 \%$ overlap, suggesting that there is limited predictability for anatomy given knowledge of electrophysiology, and vice versa. $61 \%$ of anatomic cluster 1 cells $(n=26)$ matched up with electrophysiological cluster $1(n=23)$, which in turn had $54 \%$ of its cells matched. Similarly, $69 \%$ of anatomic cluster 2 cells $(n=32)$ matched with electrophysiological cluster 2 

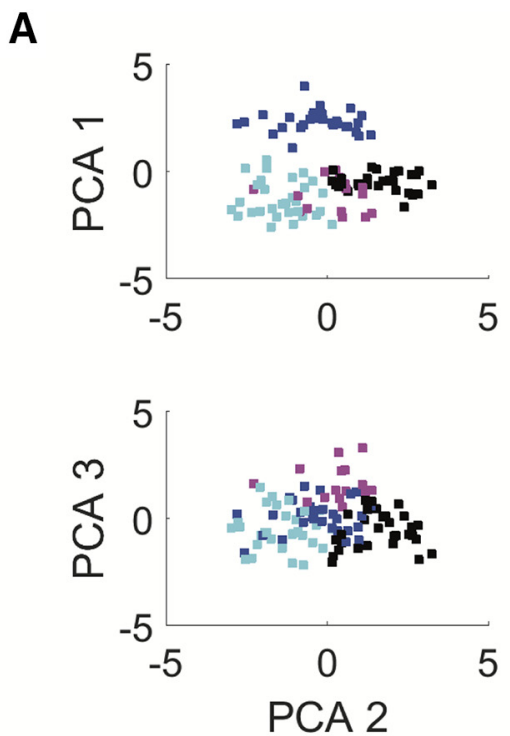

$\mathrm{Bi}$

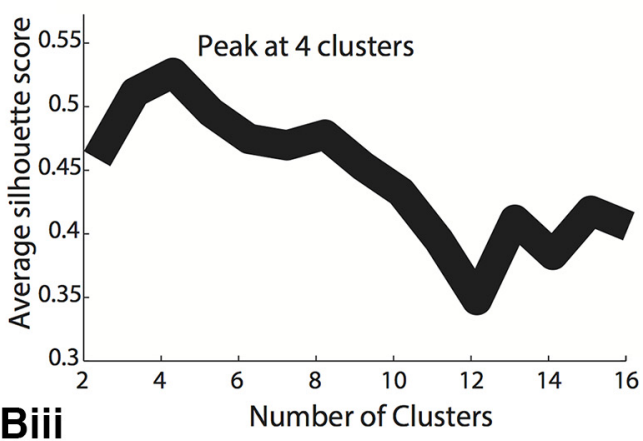

Biii

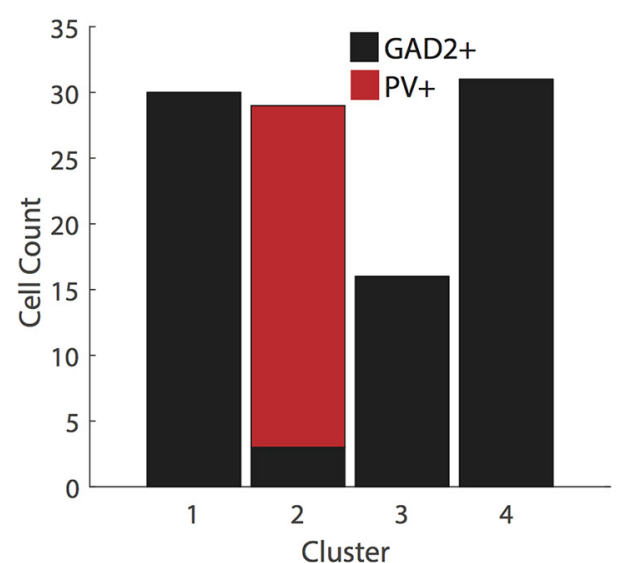

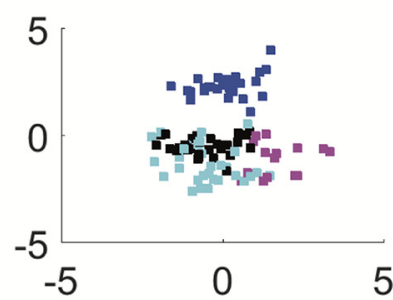
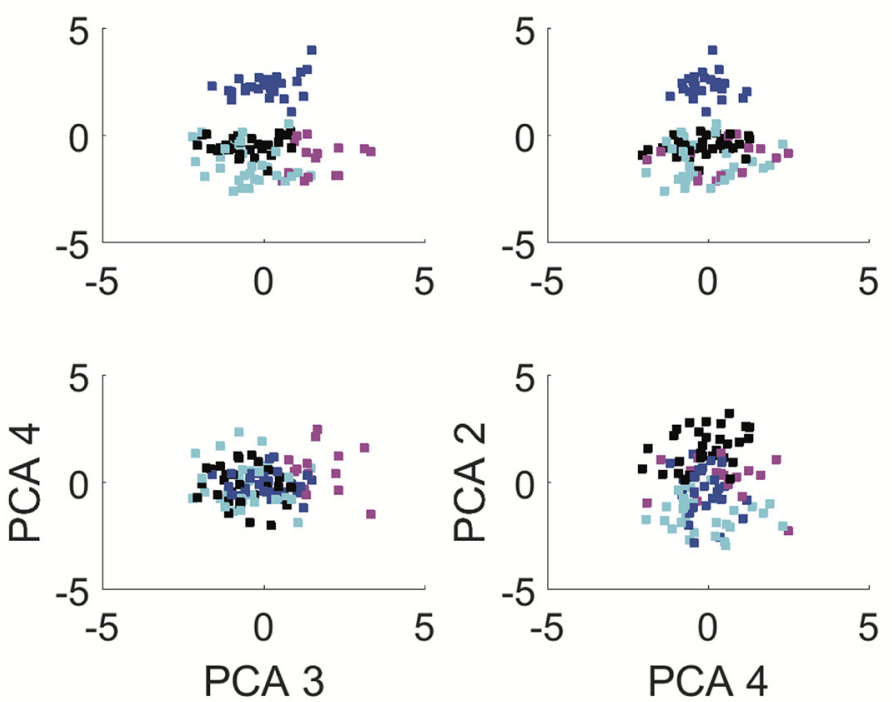
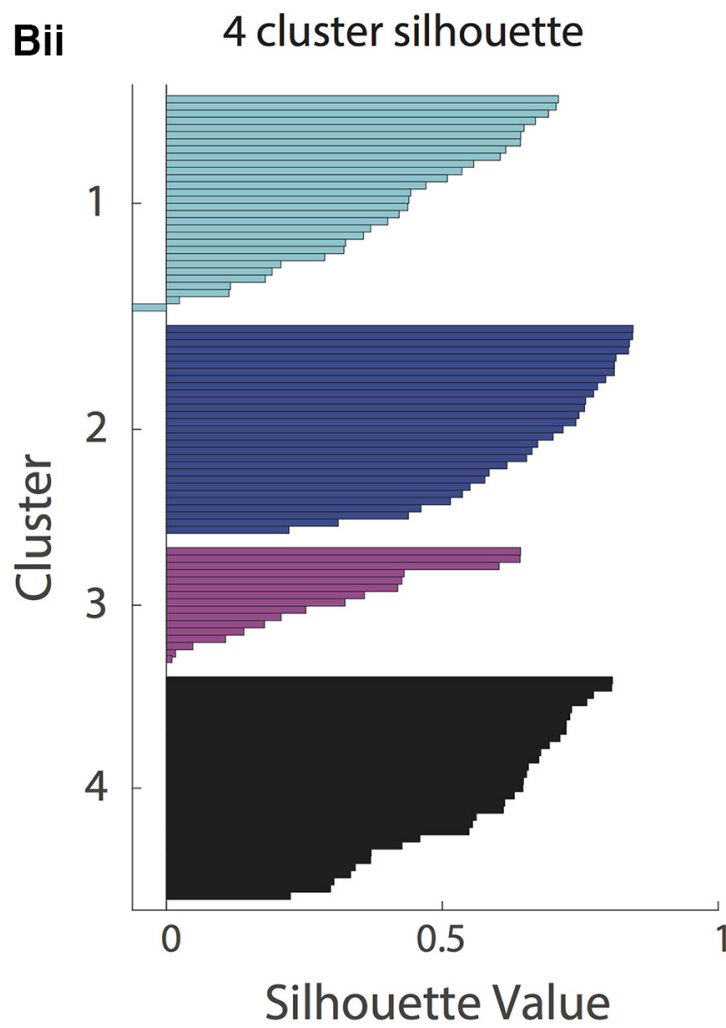

Figure 4. PCA and k-means clustering analysis. $\boldsymbol{A}$, The first four principal components of combined electrophysiological and anatomic data are plotted in all possible combinations. These four principal component dimensions were used to conduct k-means clustering analysis. Cluster 1 is cyan, cluster 2 is blue, cluster 3 is magenta and cluster 4 is in black. Bi, To determine the optimal number of clusters for k-means clustering, the average silhouette score (measure of distance for within-cluster points compared to outside-ofcluster points) was calculated for k-means clustering analyses using between 2 and 16 clusters. The highest silhouette score was achieved using four clusters, suggesting that this is the optimal cluster number. Bii, The silhouette value (score) for each point is shown in their corresponding cluster. Low or negative silhouette values indicate points that fit poorly within its cluster. Biii, In a four-cluster analysis, PV + cells were located entirely in cluster 2, with 26 out of 29 cells being PV + . This again suggests that using four clusters for the k-means clustering analysis is optimal. 
Ai Combined anatomy and electrophysiology analysis
Aii

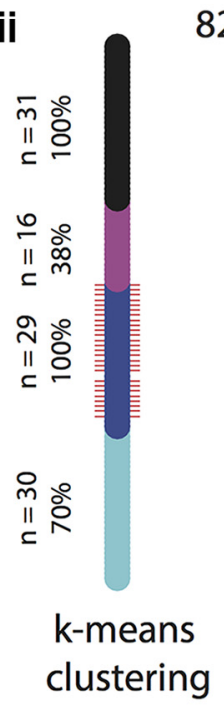

$82 \%$ overlap

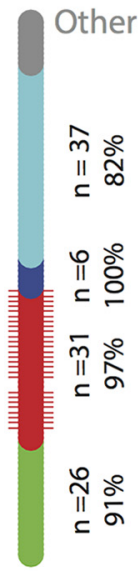

Hierarchical clustering

\section{Separate anatomy and electrophysiology analysis}
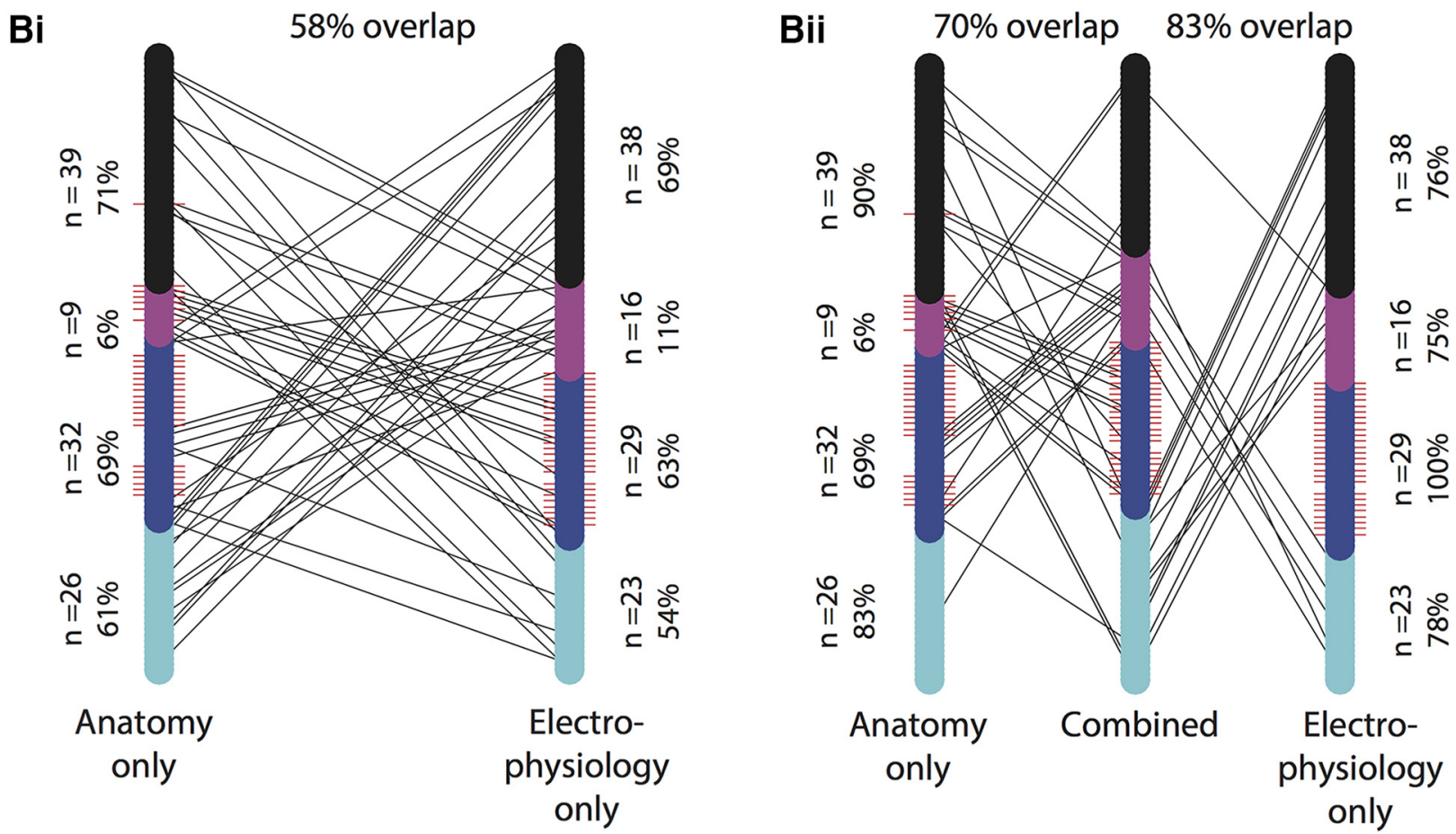

Figure 5. Comparison of different clustering methods: k-means clustering versus hierarchical clustering, combined versus separate anatomic and electrophysiological analysis. A, UPGWA hierarchical clustering using combined anatomic and electrophysiological data yielded similar results to k-means clustering. Ai, UPGWA hierarchical dendrogram separates the 106 interneurons sequentially by the least squared Euclidean distance. Each branching point represents the splitting of a cluster into two clusters, until the clusters are comprised of single neurons. Each end point thus represents a single interneuron. Branch points above the height of 2.8 (a.u.), in this case representing $52 \%$ of the maximum distance in the population, are considered to represent distinct clusters. These resulted in eight different clusters. To match up these clusters with those derived from the k-means clustering analysis, all possible permutations were tested. The permutation with maximum overlap, shown per the labels for each cluster, was used for further analysis. Aii, The clustering distribution for hierarchical clustering is shown on the left bar, with each color corresponding to the branch on the dendrogram. The four clusters with less than four interneurons were grouped into the gray "other" category. The clustering distribution for the k-means clustering is shown on the right, in the same color scheme used throughout the rest of the chapter. For each distribution, red whiskers represent the PV+ interneurons. Black lines connect corresponding interneurons that were categorized differently in each distribution, therefore fewer lines indicate greater overlap between clustering methods. The two clustering methods showed $82 \%$ overlap, meaning $82 \%$ of interneurons were categorized within the same cluster. The number of interneurons 
continued

in each cluster is noted beside each cluster, along with the percentage of that cluster which was classified into their corresponding cluster in the other clustering method. For example, cluster 1 in the hierarchical clustering method has 26 interneurons, $91 \%$ of which were also classified into cluster 1 in the k-means clustering method. $\boldsymbol{B}$, K-means clustering was used to cluster all 106 interneurons using only one type of data: either anatomic or electrophysiological. Bi, Distribution plots for purely anatomic clustering and purely electrophysiological clustering are shown as in Aii. Both anatomic and electrophysiological clustering were matched to the combined, four-cluster k-means clustering distribution, as described in Materials and Methods. The overlap between purely anatomic and purely electrophysiological clustering was $58 \%$, indicating that some, but not most, interneurons could be matched to different anatomic and electrophysiological profiles. $\mathbf{B i i}$, The same anatomic and electrophysiological distributions as in $\boldsymbol{B} \boldsymbol{i}$ are shown in comparison with the combined distribution in the center. There is a $70 \%$ overlap between the combined distribution and the anatomic, whereas there is $83 \%$ overlap between the combined and electrophysiological distribution.

$(n=29)$, of which $63 \%$ matched. Electrophysiological cluster 2 had all of the PV+ cells, which indicates that these cells could be well clustered using only electrophysiological data. Anatomic cluster 2, however, had a smaller fraction of PV $+(21$ out of $26,81 \%$ ) cells, suggesting that anatomic data were not as clear-cut a differentiator for $\mathrm{PV}+$ cells. Anatomic cluster 3 was small, with only nine cells, only $6 \%$ of which matched with electrophysiological cluster 3 . This cluster had 16 cells, only $11 \%$ of which matched with anatomic cluster 3 . Overall cluster 3 showed almost no correlation between its anatomy and its electrophysiology. Cluster 4 showed more correct matches between the anatomic $(n=39,71 \%)$ and the electrophysiological ( $n=38,69 \%)$ distributions.

We then inspected the similarity of each separate analysis to the combined distribution used in the study, as shown in Figure 5Bii. The anatomic distribution had 70\% overlap with the combined distribution, whereas the electrophysiological cluster had $83 \%$ overlap with combined distribution. Anatomic clusters were matched with their corresponding combined clusters in proportions of $83 \%$, $69 \%, 6 \%$, and $90 \%$, respectively. Electrophysiological clusters were matched with their corresponding combined clusters in proportions of $78 \%, 100 \%, 75 \%$, and $76 \%$, respectively.

\section{Description of the four interneuron groups}

Assigned groups express distinct electrophysiological/ anatomic profiles

The clustering method classified the dataset into four interneuron groups with distinct combinations of anatomic and electrophysiological profiles. Table 2 shows the average soma depth and the average axonal tree extent for each cluster. The average soma depth varies slightly among clusters, with most of the variance being within cortical layer 2. The average axonal extent, however, is distinct among clusters, with each cluster projecting to a different range of cortical layers. Table 2 also shows the electrophysiological characteristics of all the clusters. Overall, no two clusters show similar electrophysiological profiles; although for certain features two clusters may have distributions with substantial overlap, the combination of electrophysiological features for each cluster is unique.

\section{Cluster 1: layer 2/3-projecting slow-firing interneurons}

Cluster 1 interneurons $(n=30)$ have somas throughout layers 2 and 3 , with an average depth of $327.0 \pm 14.2 \mu \mathrm{m}$. Their axonal projection reach layers 2 and 3 , and their average axonal extent is the deepest of all clusters at
$296.4 \pm 21.5 \mu \mathrm{m}(p<0.01)$ for its most superficial extent and $446.4 \pm 23.0 \mu \mathrm{m}(p<0.05)$ for its deepest extent. The average axonal width is narrower $(p<0.01)$ than cluster 2 and 4 at $361.4 \pm 21.7 \mu \mathrm{m}$. Several examples of interneurons belonging to this cluster are shown in Figure 6, with all somas and axonal trees shown in Figure $6 A \mathrm{~A}$ and four reconstructions in Figure 6Bi-Biv.

Cluster 1 is similar to cluster 4 electrophysiologically, with a slow firing rate and flat F-I relationship. The interneurons in this cluster had both the lowest F-I gain at $153.0 \pm 18.9 \mathrm{~Hz} / \mathrm{nA}(p<0.01$ with respect to clusters 2 and 3) and peak firing frequency at $57.4 \pm 5.1 \mathrm{~Hz}(p<$ 0.01 with respect to clusters 2 and 3). The average input resistance of cluster 1 interneurons is $220.2 \pm 12.3 \mathrm{M} \Omega$, the second highest and significantly different from clusters 2 and $4(p<0.05)$. The mean falling (rising) time constant is $13.7 \pm 0.8 \mathrm{~ms}(14.1 \pm 0.8 \mathrm{~ms})$, significantly longer than in cluster $2(p<0.01)$ and shorter than in cluster $3(p<0.01)$ but not significantly different from neurons in cluster 4 . The resting membrane potential for cluster 1 interneurons averaged at $-66.3 \pm 1.0 \mathrm{mVm}$ more depolarized than cluster $2(p<0.01)$ but more hyperpolarized than cluster $3(p<0.01)$. The mean rheobase was $185.7 \pm 15.7 \mathrm{pA}$, lower than both clusters 2 and $4(p<0.05)$. The average lowest firing rate was $10.6 \pm 2.3$ $\mathrm{Hz}$. The mean firing threshold is $-38.7 \pm 0.9 \mathrm{mV}$, not significantly different to any other cluster. The AP rise time average for cluster 1 interneurons is $0.273 \pm 0.006 \mathrm{~ms}$, and its AP half-width is $1.085 \pm 0.034 \mathrm{~ms}$, significantly greater than in cluster $2(p<0.01)$. A sample spike shape from a cluster 1 interneuron is shown in Figure 7Aii. Cluster 1 neurons have the shallowest spike AHP of all clusters except cluster 3 at $16.2 \pm 1.0 \mathrm{mV}(p<0.01)$. They have a smaller change in impedance between $-80 \mathrm{mV}$ and the perithreshold region than clusters 2 and $3(p<0.01)$, with an average percentage change of $64.8 \pm 10.6(\%)$. The average sag ratio for the interneurons of this cluster is $0.936 \pm 0.006$. The adaptation ratio averages $0.783 \pm$ 0.153. In all, the interneurons in cluster 1 are characterized by axonal projections throughout layers 2 and 3 and the slowest firing rates of all interneuron clusters except cluster 4. The interneurons are differentiated from cluster 4 mainly by their greater input resistances and time constants.

\section{Cluster 2: layer 2/3-projecting fast-firing interneurons}

Cluster 2 is the only cluster containing PV + interneurons, which comprise 26 out of the 29 cells in this group. Somas of cells in cluster 2 are located throughout layers 

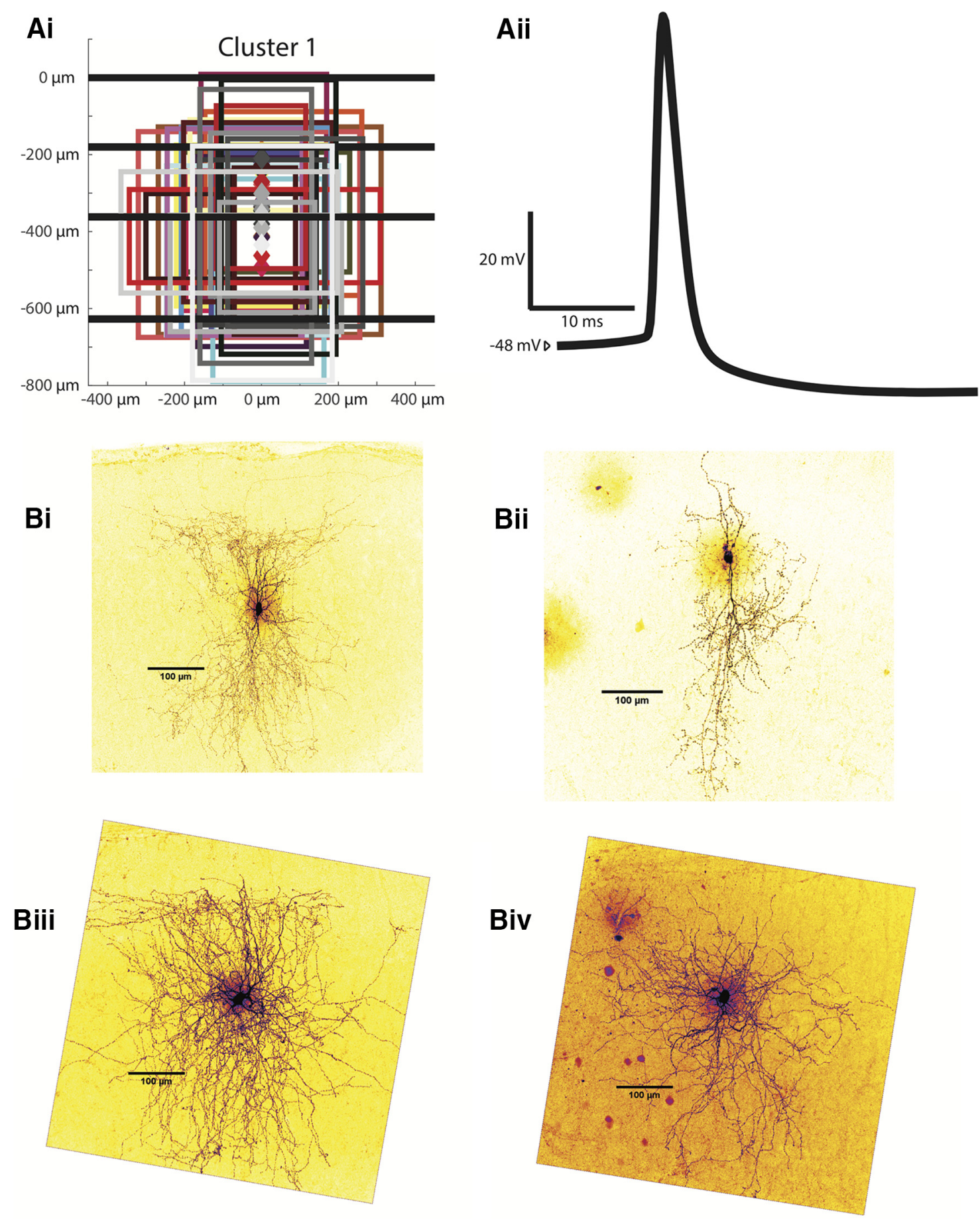

Figure 6. Examples of cluster 1 interneurons. Ai, Anatomic characteristics for all cells in cluster 1 are shown in the same abstract form as in Figure 1Bii. Aii, An example of an averaged AP from a characteristic cluster 2 interneuron. Bi-Biv, Z-stack projections of cluster 1 Alexa Fluor 488-labeled MEC interneurons are shown as examples.

2 and 3, with an average depth of $304.9 \pm 16.1 \mu \mathrm{m}$. Like those from cluster 1 , cluster 2 axonal projections are located mainly throughout layers 2 and 3 . The most superficial/deepest axonal projections are on average $168.4 \pm 19.8 \mu \mathrm{m}$ and $298.0 \pm 16.4 \mu \mathrm{m}$, respectively, which places them significantly more superficial than those from cluster 1 cells $(p<0.01)$. Average axonal width is $513.0 \pm 12.8 \mu \mathrm{m}$. Figure $7 \mathrm{Ai}$ shows all axonal trees for this cluster, and in Figure 7Bi-Biv, there are several examples of two-photon reconstructions.

Cluster 2 interneurons are predominantly fast-spiking, in agreement with previous findings on PV + cells (Jones and Bühl, 1993). The interneurons in this cluster have a very high peak firing rate of $279.2 \pm 8.6 \mathrm{~Hz}$, significantly higher than cells from all other clusters $(p<0.01)$ and $250 \%$ greater than the next highest spiking firing cluster 
$\mathrm{Ai}$

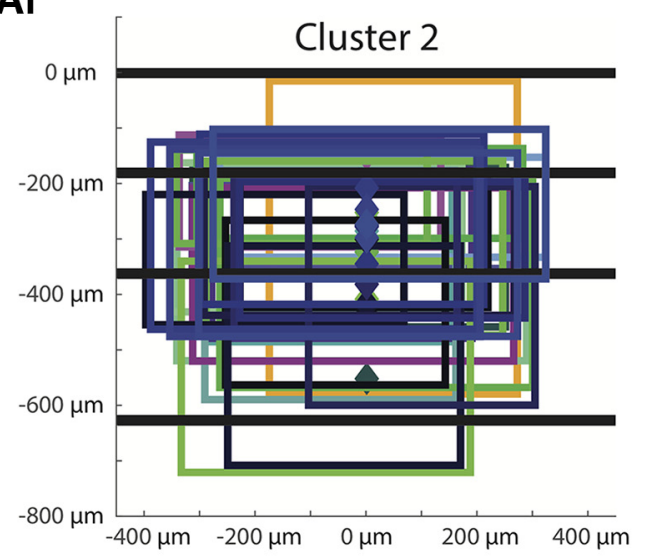

Bi
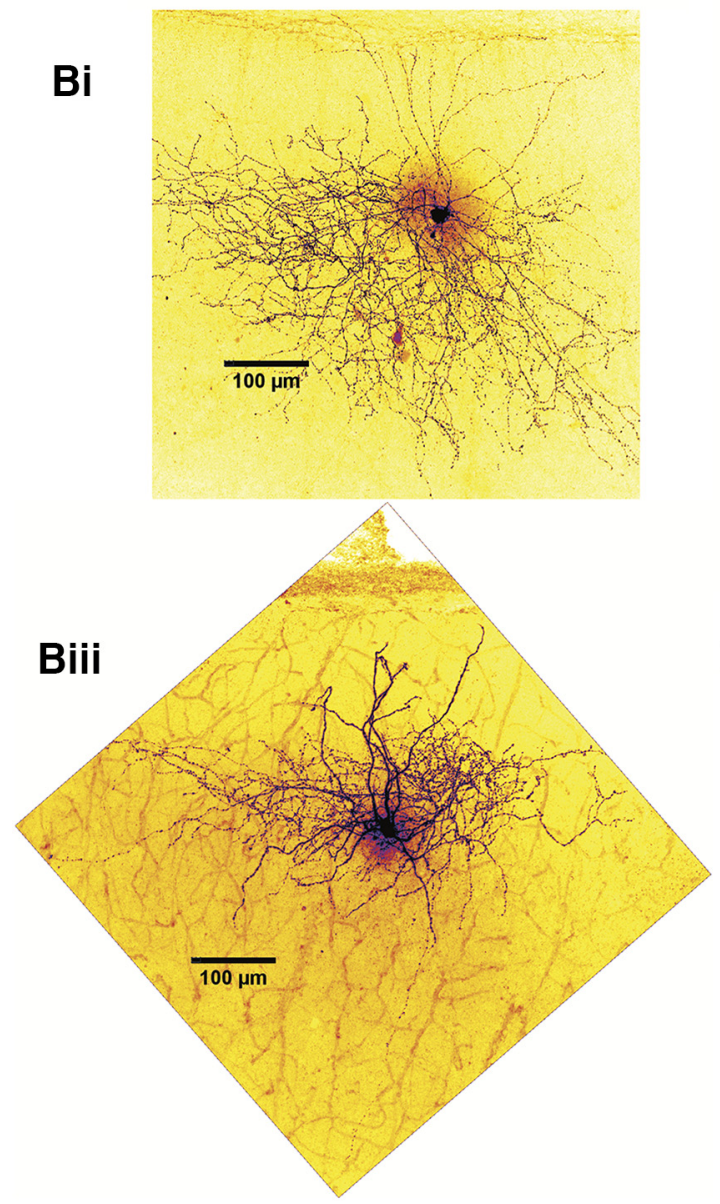
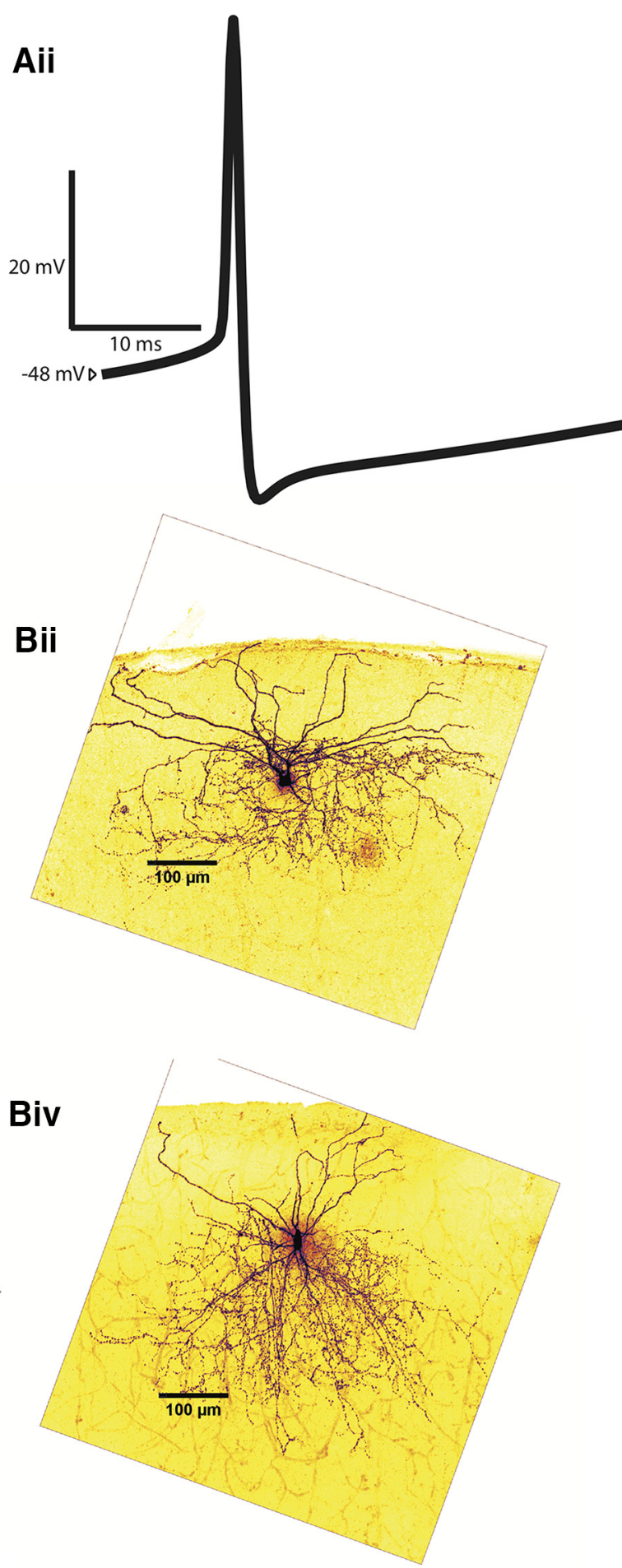

Figure 7. Examples of cluster 2 interneurons. Ai, Anatomic characteristics for all cells in cluster 2 are shown in the same abstract form as in Figure 3.1Bii. Aii, An example of an averaged AP from a characteristic cluster 2 interneuron. Bi-Biv, Z-stack projections of cluster 2 Alexa Fluor 488-labeled MEC interneurons are shown as examples.

(cluster 3 at $111.5 \pm 8.4 \mathrm{~Hz}$ ). The average input resistance of cluster 2 interneurons is $85.7 \pm 5.1 \mathrm{M} \Omega$, by far the lowest of all clusters $(p<0.01)$. The resting membrane potential for cluster 2 interneurons averaged at $-71.4 \pm$ $1.0 \mathrm{mV}$, more hyperpolarized than all other clusters $(p<$ 0.05). The mean rheobase was $380.7 \pm 22.2 \mathrm{pA}$, lower than all other clusters $(p<0.05)$. The lowest firing frequency is significantly higher than all other clusters at $79.1 \pm 8.6 \mathrm{~Hz}(p<0.01)$. The falling (rising) time constant is also smaller than all other clusters $(p<0.01)$ at $5.1 \pm$ $0.2 \mathrm{~ms}(5.2 \pm 0.2 \mathrm{~ms}) ;$ as are the AP half-width $(0.526 \pm$ $0.010 \mathrm{~ms}, p<0.01)$ and the AP rise time $(0.192 \pm 0.003$, 
$p<0.01$ ). A sample spike shape from a cluster 2 interneuron is shown in Figure 7 Aii. Its $19.9 \pm 0.5 \mathrm{mV}$ spike AHP is similar to those from clusters 3 and 4 , but significantly greater than AHPs from cluster $1(p<0.05)$. Despite its fast firing rate, the F-I gain is only the second highest of the four clusters at $281.9 \pm 15.8 \mathrm{~Hz} / \mathrm{nA}$, greater than in cluster 1 and 4 ( $p<0.01$ for all comparisons). The percentage change in impedance exhibited in cluster 2 neurons is $133.1 \pm 12.7(\%)$, similar to results from cluster 3 but greater than those in clusters 1 and $4(p<0.01)$. The average sag ratio is $0.945+0.004$, and the adaptation ratio is $0.878 \pm 0.007$, significantly larger than in cluster 1 $(p<0.01)$ and less than clusters $3(p<0.01)$ and $4(p<$ 0.05). The key features of the interneurons of cluster 2 are expression of PV, axonal projections throughout layers 2 and 3 , the most hyperpolarized resting membrane potential, and the fastest firing rate and lowest input resistance of all interneuron clusters.

\section{Cluster 3: layer 1/2-projecting interneurons}

Cluster 3 interneurons $(n=16)$, like the first two clusters, have somas throughout layers 2 and 3 . The average soma depth in cluster 3 is $274.6 \pm 19.7 \mu \mathrm{m}$. Cluster 3 axonal projections extend mainly through layers 1 and 2 . The average axonal extent of cluster 3 interneurons is similar to that from cluster 2 interneurons at $107.0 \pm 33.2$ $\mu \mathrm{m}$ in its superficial extent and $318.0 \pm 35.6 \mu \mathrm{m}$ in its deep extent (more superficial than in cluster $1, p<0.05$ ). Its anatomic profile is not significantly different from cluster 2 in any respect. Its axonal tree average is $440.6 \pm$ $30.8 \mu \mathrm{m}$. Cluster 3 is the smallest cluster, having only 16 cells. All the axonal trees for this cluster are shown in Figure $8 \mathrm{Ai}$, and several two-photon reconstruction examples are shown in Figure 8Bi-Biv.

Cluster 3 interneurons have the highest input resistance $(274.4 \pm 14.9 \mathrm{M} \Omega)$ of any cluster $(p<0.01)$ except cluster 1. Cluster 3 interneurons also have the steepest F-I gain of all clusters except cluster 2 at $373.9 \pm 51.4 \mathrm{~Hz} / \mathrm{nA}(p<$ $0.01)$. The average falling (rising) time constant is $15.8 \pm$ $0.9 \mathrm{~ms}(16.2 \pm 1.0 \mathrm{~ms})$, greater than clusters 1 and $2(p<$ $0.01)$. The resting membrane potential for cluster 3 interneurons averaged at $-55.5 \pm 2.1 \mathrm{mV}$, more depolarized than all other clusters $(p<0.01)$. The mean rheobase was $118.8 \pm 12.3 \mathrm{pA}$, significantly lower than clusters 2 and $3(p<0.01)$. The firing threshold is $-40.8 \pm 1.1 \mathrm{mV}$, more hyperpolarized than clusters 2 and $4(p<0.05)$. The AP half-width $(0.832 \pm 0.067 \mathrm{~ms})$ is significantlygreater than cluster $2(p<0.01)$ but less than cluster 1 and $4(p<$ 0.05 ). The AP rise time is $0.253 \pm 0.013 \mathrm{~ms}$, and the spike AHP $19.3 \pm 1.0 \mathrm{mV}$. A sample spike shape from a cluster 3 interneuron is shown in Figure 8Aii. The peak firing rate is $111.5 \pm 8.4 \mathrm{~Hz}$, higher than cluster 1 but less than cluster $2(p<0.01)$. The lowest firing rate is $13.6 \pm 2.7 \mathrm{~Hz}$. The change in impedance for cluster 3 is $136.5 \pm 15.7$ (\%). Cluster 3 has the lowest sag ratio at $0.888 \pm 0.013$ $(p<0.05)$, the only sag ratio to be significantly different compared to other clusters. The average adaptation ratio was $1.335 \pm 0.730$, significantly greater than cluster 2 $(p<0.05)$. Cluster 3 interneurons are defined by their axonal projections restricted mainly to layer 1 and 2 , as well as having the most depolarized resting membrane potential and a relatively high input resistance and F-I gain.

\section{Cluster 4: layer 1-projecting interneurons}

Cluster 4 interneurons $(n=31)$ have the most superficial somas of any cluster $(p<0.01)$. At an average soma depth of $191.6 \pm 8.9 \mu \mathrm{m}$, cluster 4 interneuron somas are located throughout layers 1 and 2 . This cluster's axonal projections are mainly limited to layer 1 , with its average deepest axonal extent being the most superficial of than cluster $1(252.9 \pm 25.7 \mu \mathrm{m}, p<0.01)$. The average most superficial axonal extent is $114.0 \pm 25.2 \mu \mathrm{m}$ and the axonal width is $472.2 \pm 20.1 \mu \mathrm{m}$. The entire population of somas and axonal extents is shown in Figure $9 A \mathrm{~A}$, and several examples of two-photon reconstructions for cluster 4 interneurons are shown in Figure 9Bi-Biv.

Most of the electrophysiological features for these layer 1-projecting interneurons do not lie at either extreme among the clusters. Input resistance is $155.1 \pm 6.2 \mathrm{M} \Omega$, greater than in cluster $2(p<0.01)$ but less than in clusters $1(p<0.05)$ and $3(p<0.001)$. The resting membrane potential for cluster 4 interneurons averaged at $-66.8 \pm$ $1.1 \mathrm{mV}$, between clusters 2 and $3(p<0.05)$. The mean rheobase was $262.6 \pm 13.2 \mathrm{pA}$, greater than clusters 1 and $3(p<0.05)$ but less than cluster $2(p<0.05)$. The average $\mathrm{F}$-I gain is $162.0 \pm 13.0 \mathrm{~Hz} / \mathrm{nA}$, less than clusters 2 and $3(p<0.01)$, Average peak firing rate is $75.3 \pm 5.6$ $\mathrm{Hz}$, less than in cluster $2(p<0.01)$. The average lowest firing frequency is $13.9 \pm 2.3 \mathrm{~Hz}$, similar to that of cluster 1 and 3 . The cluster 4 average falling (rising) time constant is $8.9 \pm 0.4 \mathrm{~ms}(9.5 \pm 0.4 \mathrm{~ms})$, and firing threshold is $-35.6 \pm 1.0 \mathrm{mV}$. The AP rise time is $(0.292 \pm 0.009 \mathrm{~ms})$. The AP half-width is $1.151 \pm 0.047 \mathrm{~ms}$, greater than those of clusters $2(p<0.01)$ and $3(p<0.05)$. A sample spike shape from a cluster 4 interneuron is shown in Figure 9Aii. The spike AHP is $20.8 \pm 0.7 \mathrm{mV}$. The percentage change in impedance is $51.6 \pm 9.2(\%)$, lower than clusters 2 and $3(p<0.01)$. Finally, the sag ratio for cluster 4 is $0.936 \pm$ 0.008 , and the adaptation ratio is $0.917 \pm 0.153$. Overall, cluster 4 interneurons are characterized by their superficial somas and axonal projections that are relatively limited to layer 1 , with electrophysiological features at neither extreme among the interneuron clusters.

\section{Discussion}

Having systematically characterized 106 interneurons in the superficial MEC, we have found that this interneuron population is best classified into four distinct groups, based on their anatomic and electrophysiological characteristics. In anatomic classifiers, the laminar extent of axonal projection and the somatic depth of interneurons were emphasized. For electrophysiological classification, input resistance, peak firing rate, rising time constant, change in impedance and F-I gain were used. The resulting interneuron groups are layer 2/3-projecting, slowfiring neurons; layer 2/3-projecting, fast-firing neurons (mainly PV+); layer 1/2-projecting interneurons; and layer 1 -projecting interneurons. The anatomic and electrophysiological characteristics of each of the interneuron groups are described in Table 2 and summarized in Figure 10. 
Ai

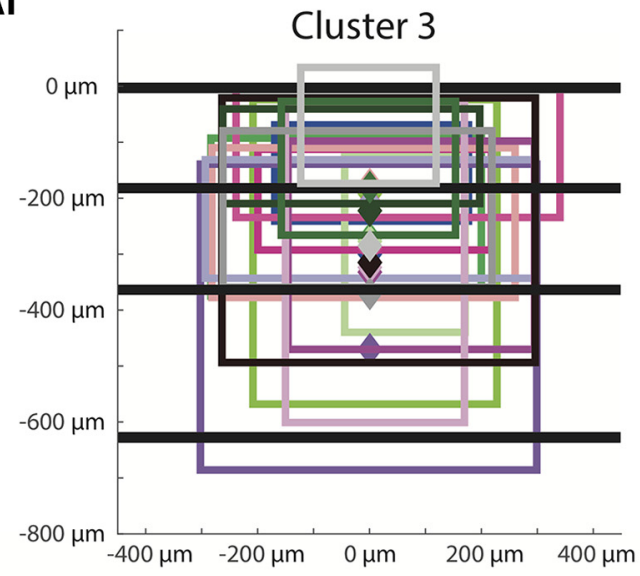

Bi

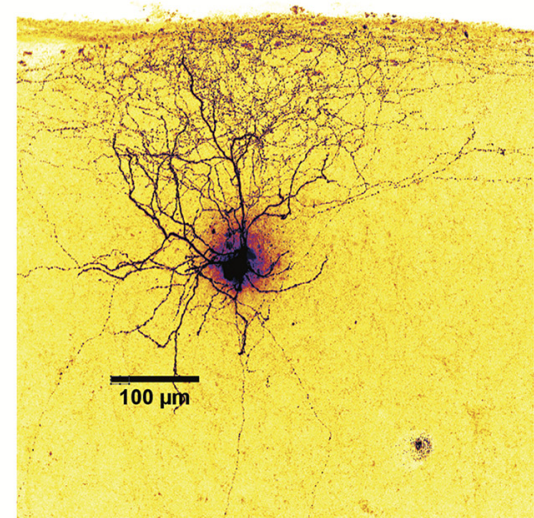

Biii

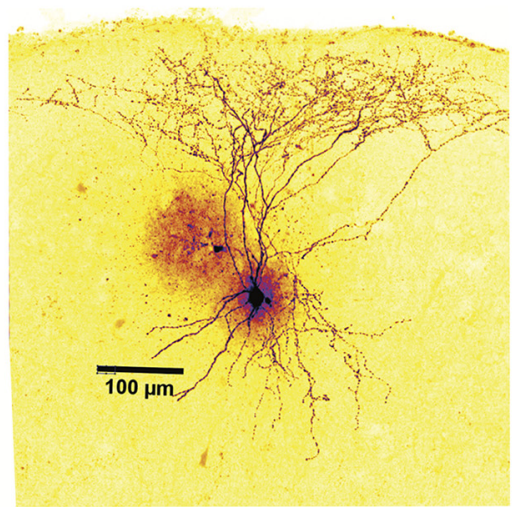

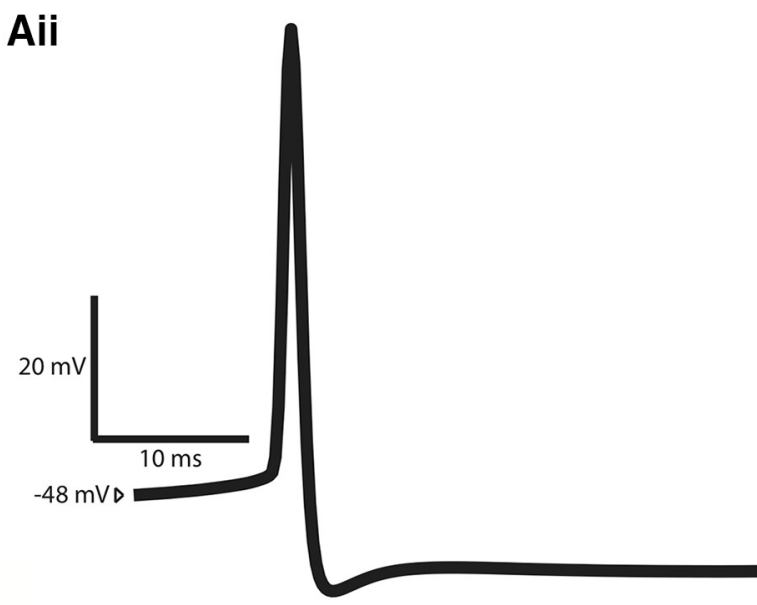

Bii

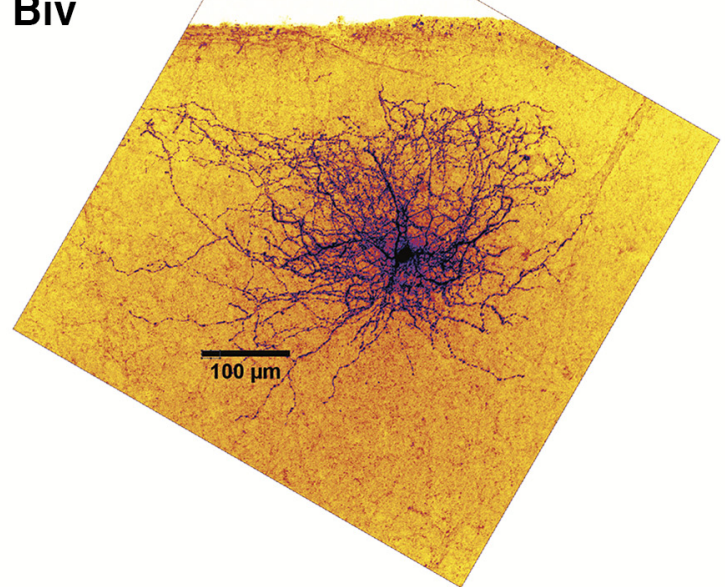

Figure 8. Examples of cluster 3 interneurons. Ai, Anatomic characteristics for all cells in cluster 3 are shown in the same abstract form as in Figure 3.1Bii. Aii, An example of an averaged AP from a characteristic cluster 3 interneuron. Bi-Biv, Z-stack projections of cluster 3 Alexa Fluor 488-labeled MEC interneurons are shown as examples.

\section{Clustering anatomic and electrophysiological data}

The clustering method for interneuron data used PCA to reduce the nine-dimensional parameter space into four orthogonal dimensions (principal components) with maximized variance (Jolliffe, 2002). Multidimensional clustering was then performed on the first four principal components and silhouette scores were used to determine optimal cluster number. This method is similar to previous approaches to neuronal classification (Cauli et al., 2000; Krimer, 2005; Dumitriu et al., 2007; Helm et al., 2013). This study is the first, to our knowledge, to combine both anatomic and electrophysiological characteristics in the 
Ai

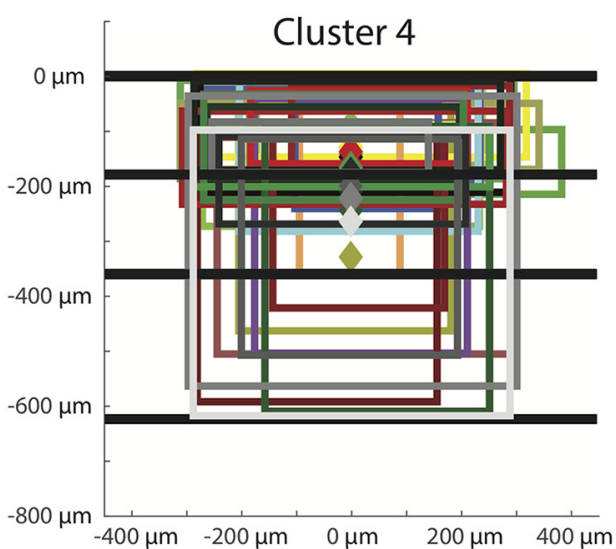

Bi

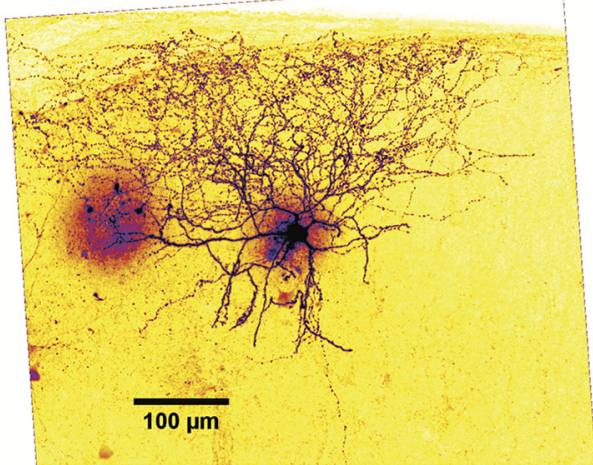

\section{Biii}

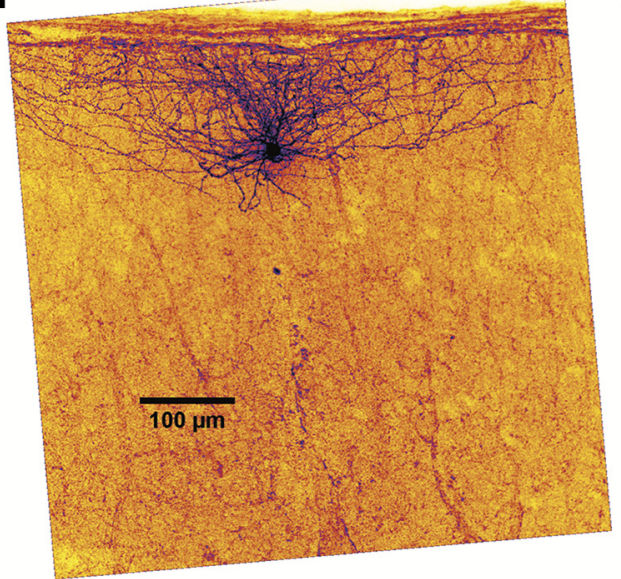

Aii

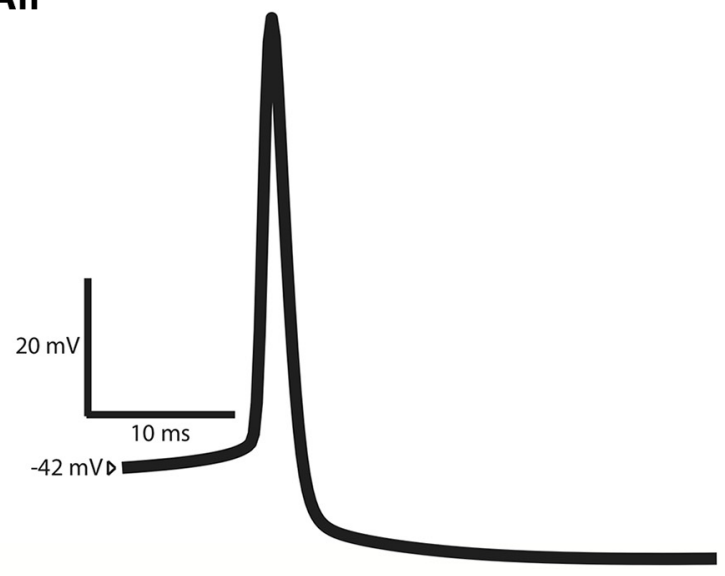

Bii

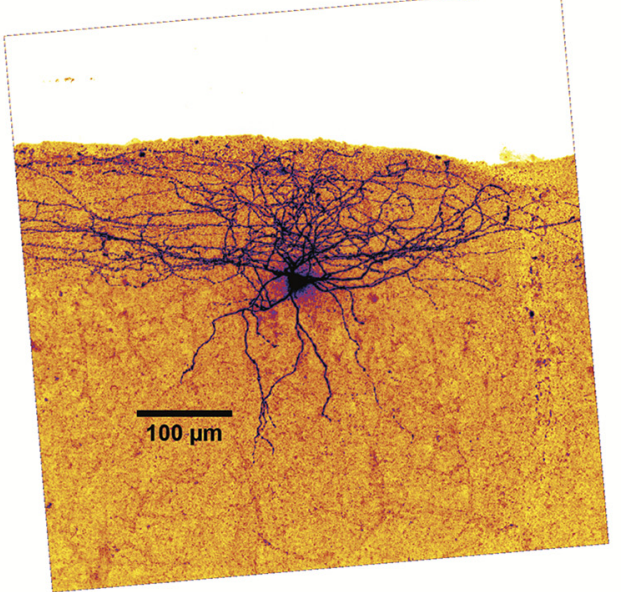

Biv

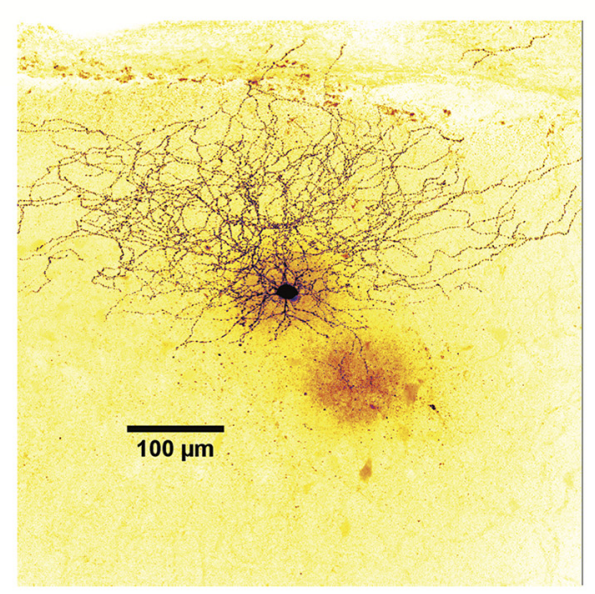

Figure 9. Examples of cluster 4 interneurons. Ai, Anatomic characteristics for all cells in cluster 4 are shown in the same abstract form as in Figure 3.1Bii. Aii, An example of an averaged AP from a characteristic cluster 4 interneuron. Bi-Biv, Z-stack projections of cluster 4 Alexa Fluor 488-labeled MEC interneurons are shown as examples.

analysis, as opposed to using only electrophysiological data (Krimer, 2005; Helm et al., 2013) or conducting clustering analyses for different types of data separately and evaluating correlations (Cauli et al., 2000; Dumitriu et al., 2007). This method is often used to differentiate between cell types within distinct molecular subgroups, such as PV+ or somatostatin (SOM) + interneurons (Hala- bisky, 2006; Ma, 2006; McGarry, 2010). Our dataset included cells from the general GAD2+ population and the specific molecular $\mathrm{PV}+$ subgroup, and the clustering method was capable of differentiating the two populations using only electrophysiological and anatomic characteristics.

Anatomic classification used axonal properties and excluded dendritic properties. Our approach was based on 
Ai

layer 2/3 projecting, slow firing

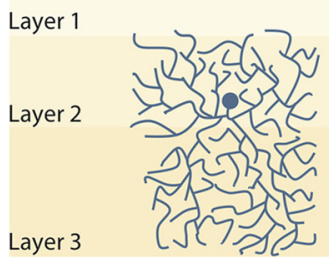

Bi

layer 2/3 projecting, fast firing

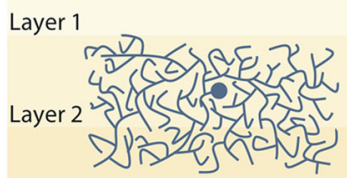

Layer 3

\section{Ci}

layer $1 / 2$ projecting

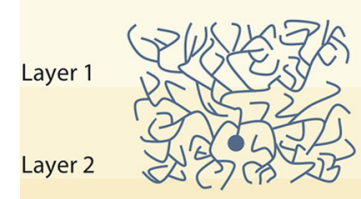

Layer 3

Di layer 1 projecting

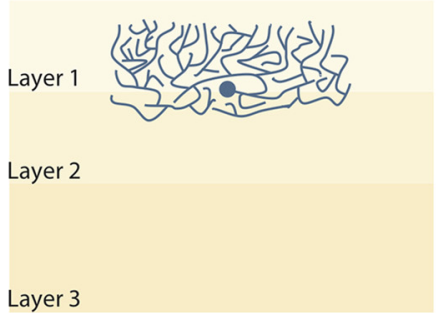

Aii
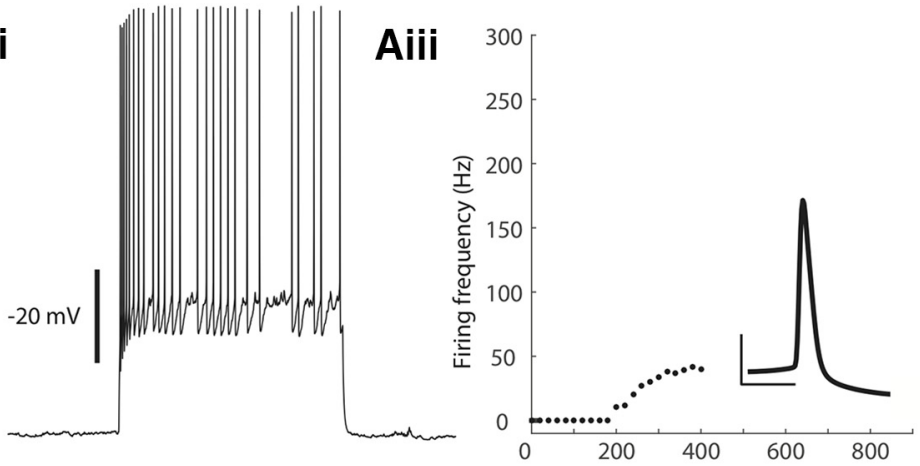

Bii
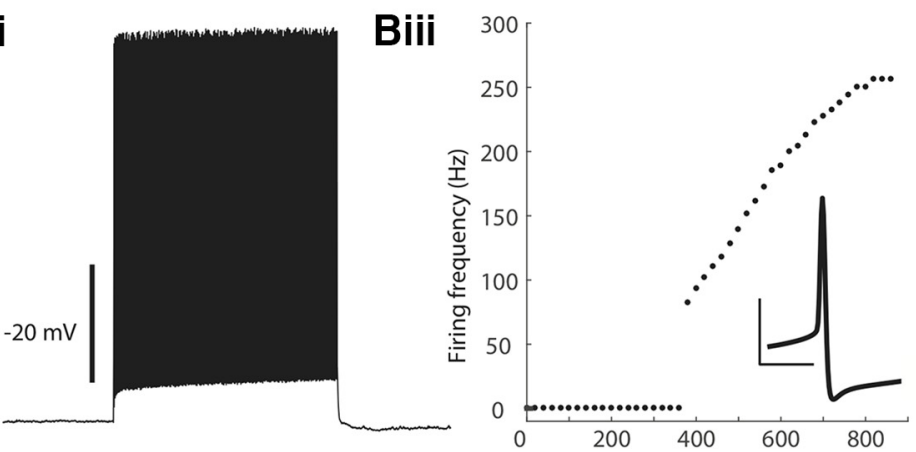

\section{Cii}

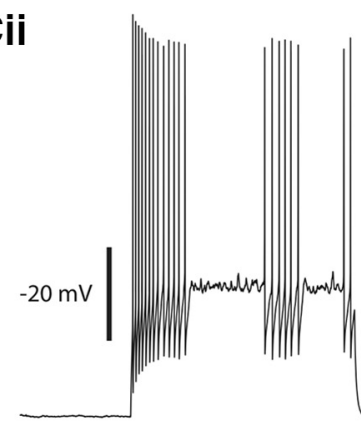

Ciii

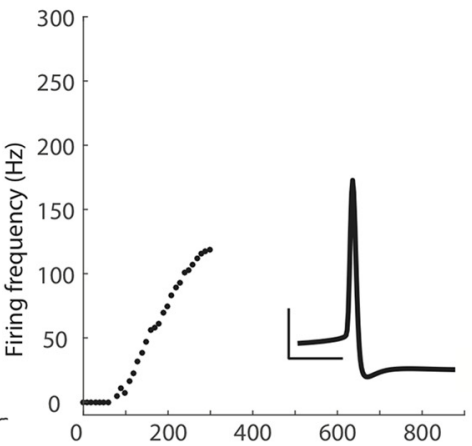

Dii

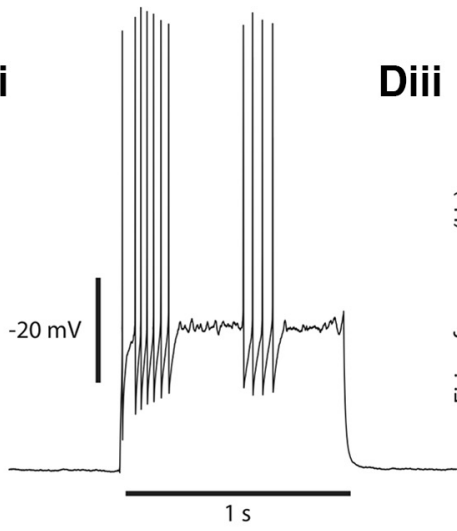

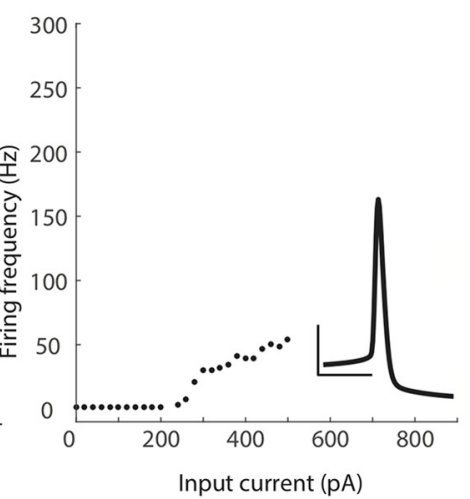

Figure 10. Interneuron groups of the superficial MEC. $\boldsymbol{A}$, Layer $2 / 3$ projecting, slow firing interneurons. $\boldsymbol{A} \boldsymbol{i}$, Representations of the average soma depth and axonal projections are shown. Aii, A 1-s spike train of a representative cell. Aiii, A typical F-I curve for this population, with the inset showing an average AP (vertical scale bar is $20 \mathrm{mV}$ and horizontal scale bar is $10 \mathrm{~ms}$ ). $\boldsymbol{B}$, Layer $2 / 3$ projecting, fast firing interneurons. Bi-Biii, Same as $\boldsymbol{A}$. C , Layer 1/2 projecting interneurons. Ci-Ciii, Same as $\boldsymbol{A}$. $\boldsymbol{D}$, Layer 2 projecting interneurons. Di-Diii, Same as $\boldsymbol{A}$. 
the clustering system for GABAergic interneurons proposed in DeFelipe et al. (2013). This system places greater importance in the location and spread of axonal arborization of GABAergic interneurons for the purpose of classification. Axonal projections form the basis for circuit connectivity and thus basing interneuronal classification on axonal geometry provides a pragmatic solution to describe the consequential anatomic characteristics of interneurons, avoiding the complexities introduced by including dendritic structure.

Two distinct methods of unsupervised clustering were used in the study, k-means clustering and hierarchical clustering. The k-means method produces independent groupings with no explicit relationship between the different clusters. To yield the optimal grouping, the k-means method requires several trials randomizing initialization conditions. The hierarchical method measures the relationship between each cell in the dataset and iteratively groups them into larger and larger groupings. Unlike the k-means clustering method, hierarchical clustering produces interrelated groupings and does not require predetermining the number of groupings in the dataset. In this study, the results of the k-means clustering were compared to those of hierarchical clustering to check whether the limitations of k-means clustering were significantly affecting the grouping outcome. Hierarchical clustering showed substantial similarity with k-means clustering, with an overlap of $82 \%$ between both analyses. This result lends support to the k-means clustering method used in the study, as similar results could be obtained using a different clustering method without the above-mentioned limitations. It is important to note that the hierarchical clustering method produces seven clusters, as opposed to four, which meant that six of the 106 interneurons were not matched to corresponding k-means clusters. This set a ceiling of $94 \%$ on the possible overlap between the two distributions.

Anatomic and electrophysiological clustering comparisons suggest there is only limited (58\%) overlap between the separate anatomic and electrophysiological profiles of the interneuron population. Cluster 1 was in both distributions the largest cluster and showed higher than average amount of overlap. Cluster 2, as the cluster containing many PV + cells, also showed higher overlap than average. Cluster 3, however, showed close to no overlap. The greater disparity in cluster size in both the anatomic and electrophysiological distributions suggest that these datasets do not conform particularly to the division into four clusters, but rather may be better fit to three clusters. This result itself suggests that while combining the two data types yields four distinct profiles of interneurons, anatomy or electrophysiology alone would not predict the same number of clusters. Electrophysiological clustering grouped all but one PV + cell into the same cluster; however anatomic clustering had six PV+ cells assigned to other clusters. Electrophysiology, thus, may be a more reliable predictor of PV expression than anatomy. When comparing the separated analyses to the combined analysis distribution, we observed that the anatomic distribution had a $70 \%$ overlap with the combined distribution, whereas the electrophysiological distribution had a $83 \%$ overlap.

\section{GAD2+ and PV+ populations}

Previous immunostaining work has found that PV + interneurons make up $\sim 50 \%$ of the GAD + population in the superficial MEC (Miettinen et al., 1996). However, analysis of the GAD2 + interneurons characterized in this cluster found a very small percentage of interneurons exhibiting characteristic PV+ electrophysiological/anatomic profiles. The clustering analysis yielded only three interneurons out of 96 that were taken to be anatomically and electrophysiologically similar to PV + interneurons by being placed in cluster 2 . This discrepancy may be explained by issues in the transgenic technique used in this study. PV + cells may also have been preferentially lost during slicing as compared with GAD2+ cells. GAD2+/ tdTomato fluorescence in PV+ neurons may have been lower than in neighboring cells, discouraging patching of $\mathrm{PV}+$ cells. Fortunately, the addition of separate PV+ transgenic animals into the study compensated in part for the relative paucity of $\mathrm{PV}+$ in the GAD2 + patched cell population.

\section{Cluster 1}

The interneurons of cluster 1 have somas located throughout layers 2 and 3 ; their axonal projections reach into layers 2 and 3 , with some neurons having axons projecting into the lamina dessicans (layer 4). Previous anatomic studies have identified MEC layer 2/3 interneurons with similar anatomic characteristics as pyramidallooking interneurons (Kumar and Buckmaster, 2006), multipolar cells (Gloveli et al., 1997), and bipolar cells (Wouterlood et al., 2000). Pyramidal-looking interneurons in the MEC layer 3 described by Kumar and Buckmaster (2006) have axonal projections mostly concentrated around the cell body in layer 3 and projecting superficially in layer 2, a feature present in some cluster 1 cells (Fig. $7 A)$. They are described as having high input resistance (382 $\pm 47 \mathrm{M} \Omega$ ), whereas the population average for cluster 1 neurons is also high relative to other clusters $(220.2 \pm 12.3 \mathrm{M} \Omega$ ). Gloveli et al. (1997) in turn described pyramidal-looking interneurons in MEC layer 3 as having much lower input resistances of $50.6 \pm 5 \mathrm{M} \Omega$, although they maintained their previously mentioned layer $2 / 3$ axonal projections. The relatively low input resistances measured by Gloveli et al. (1997) are likely due to their use of sharp electrodes (as opposed to the patch electrodes used in this study), which have been shown to reduce the input resistance in a cell by $20-40 \%$ (Li, 2004). Overall, these results suggest that a significant portion of cluster 1 cells are pyramidal-looking interneurons. Multipolar cells are described similarly by Gloveli et al. (1997), with a low input resistance of $36.8 \pm 3.3 \mathrm{M} \Omega$. Unlike the pyramidallooking interneurons, the axonal projections of these interneurons project further into layer 2 and can project onto layer 1 , in addition to projecting intralaminarly in layer 3 . This cell type contains SOM+ and cholecystokinin (CCK) + cells (Wouterlood and Pothuizen, 2000), and like the pyramidal-looking interneuron is also likely represented within the cluster 1 population. Finally, MEC layer 
3 bipolar cells described by Wouterlood et al. (2000) may be included in the cluster 1 population as the cells having narrower axonal widths that can project deeper into the lamina dessicans. Cluster 1 neurons account for the superficial MEC's deeper-projecting interneurons that generally have lower firing rates and $\mathrm{F}-\mathrm{I}$ gains. This differentiates them from the fast-firing layer 2/3-projecting interneurons and suggests that they play different roles in local circuit modulation.

\section{Cluster 2}

The second cluster described in this study is made up almost entirely of PV+ interneurons. The four out of 30 cells that are not verified to be $\mathrm{PV}+$ may indeed be $\mathrm{PV}+$, as the GAD2 + marker also covers the PV + cell population (Miettinen et al., 1996). In the MEC, the population of $\mathrm{PV}+$ neurons with somas located in layer 2 (as is the case with most cluster 2 neurons) contains basket cells and chandelier cells (Canto et al., 2008). Basket cells in the MEC were first described by Jones and Bühl (1993), who through unaided patching over several years successfully characterized 12 basket cells, both anatomically and electrophysiologically. In the anatomic description, they described cells with axonal projection mostly within layer 2 , as we see for cluster 2 neurons. Electrophysiologically, they described the PV+ interneurons as fast-spiking, and cluster 2 neurons are the fastest spiking population in the present corpus. Additionally, the basket-like interneurons had AP half-widths of $0.51+0.05 \mathrm{~ms}$, very similar to the AP half-widths of cluster 2 neurons of $0.526+0.010 \mathrm{~ms}$. Finally, the cells in cluster 2 were very likely to exhibit type 2 F-I relationships as shown by having a significantly higher lowest firing frequency than all other clusters. The large minimum firing rate discontinuity is often associated with fast-spiking PV+ cells (Mancilla et al., 2007). These cells are likely to make up the bulk of the cluster 2 interneuron population. MEC horizontal chandelier cells, named for their vertically oriented axonal aggregations, have been described having a vertical axonal extent 100$200 \mu \mathrm{m}$ long (cluster 2 average is $\sim 120 \mu \mathrm{m}$ ); the horizontal extent is usually $250-350 \mu \mathrm{m}$ wide (cluster 2 average is $513.0 \pm 12.8 \mu \mathrm{m}$, although some are narrower than 350 $\mu \mathrm{m}$; Soriano et al., 1993). By visual inspection, chandelier cells comprise a smaller fraction of the cells in cluster 2 than basket cells.

Clusters 1 and 2 have similar anatomic distributions (axonal projections mainly in layers 2 and 3 ) and so are mainly distinguished by their temporal dynamics. Cluster 1 cells have lower firing rates, lower F-I gains and larger time constants than cluster 2 cells. What role might these two interneuron populations play in the superficial MEC? First, fast-firing PV + neurons like those in cluster 2 have been shown previously to mediate stellate-to-stellate cell connectivity (Couey et al., 2013), provide grid cell-driven recurrent inhibition to the local circuit (Buetfering et al., 2014), and drive theta-nested gamma oscillations (Pastoll et al., 2013). Second, cortical circuits throughout the brain receive a large dynamic range of excitatory inputs, input which is then balanced by an increase in inhibitory inputs (Borg-Graham et al., 1998; Monier et al., 2003; Wehr and
Zador, 2003). This coordination occurs over a large dynamic range, meaning the inhibitory dynamics of each circuit is capable of matching excitatory input across this same temporal range. The existence of slow-firing (cluster 1) and fast-firing (cluster 2) inhibitory interneurons with axonal projections within the same layers may thus serve to provide enough sensitivity and dynamic range to address the heterogeneous multimodal inputs that the MEC receives, facilitating the spatial navigation functions that have been described in layers 2 and 3 . Third, optogenetic stimulation of either the PV + cell populations (as in cluster 2) and SOM+ populations (as are likely present in cluster 1) have been shown to produce ictal discharges in vitro in the superficial MEC (Yekhlef et al., 2015). Kumar and Buckmaster (2006) also showed that rats treated with pilocarpine showed reduced levels of these two cell types, which directly resulted in hyper-excitability of layer 2 stellate cells. Dysfunction of cells within clusters 1 and 2 may thus play an important role in epilepsy.

\section{Cluster 3}

Cluster 3 interneuron somas are mainly located in the layer 2 somas and have axonal projections into layers 1 and 2. Anatomic studies have described MEC and lateral entorhinal cortex cells with similar anatomic characteristics as multiform neurons, with axons similarly projecting into the white matter (layer 1) and intralaminarly in layer 2 (Tahvildari and Alonso, 2005). Electrophysiological characterization of these cells in the LEC by Tahvildari and Alonso (2005) showed cells with similar time constants $(15.8 \pm 0.9 \mathrm{~ms}$ in this study, where they showed $20.7 \pm$ $1.32 \mathrm{~ms})$ and peak firing rates $(111.5 \pm 8.4 \mathrm{~Hz}$ compared to $\sim 125 \pm 30 \mathrm{~Hz}$ ). The average firing threshold they measured in the LEC was slightly more depolarized $(-45.8 \pm 0.5 \mathrm{mV})$ than that measured in this study in the MEC $(-40.8 \pm 1.1 \mathrm{mV})$; input resistance was also considerably lower in the LEC $(55.7 \pm 6.85 \mathrm{M} \Omega)$ than in the MEC $(274.4 \pm 14.9 \mathrm{M} \Omega)$. However, their study used sharp electrodes which introduce leak conductances to the cell membrane ( $\sim 6 \mathrm{M} \Omega$ vs $80-120 \mathrm{M} \Omega$ ). The cells of cluster 3 may therefore be related to the multiform cells electrophysiologically characterized in the LEC and anatomically described in the MEC, although to our knowledge never previously described electrophysiologically. Being the cluster with the smallest sample size and most heterogeneous anatomic distribution, it is difficult to ascertain what role cluster three interneurons may play in the MEC. They have the second fastest peak firing rate and steepest F-I gain to the PV+ cluster 2 cells. Given that cluster 3 and cluster 4 both project into layer 1 , the relatively slower firing rate of cluster 4 cells suggest that these two populations play the same fast/slow complementary role that clusters 1 and 2 play in layers 2 and 3, increasing the range of inhibitory responses available to respond to excitatory inputs.

Neurons in both cluster 2 and cluster 3 show an increase in impedance as they approach threshold, averaging approximately at $35 \%$ increase from rest to the subthreshold. This phenomenon has been described in Economo et al. (2014), and may be due to a persistent 
sodium conductance that is activated as the cell is depolarized in the subthreshold regime. The presence of this effect suggests that inputs to cluster 3 and 4 neurons are amplified if they arrive when the membrane potential is near threshold.

\section{Cluster 4}

The fourth cluster describes cells with somas in layer 1 (near the layer $1 / 2$ border) and axonal projections mostly restricted to layer 1 with a horizontal extent on average $472.2 \pm 20.1 \mu \mathrm{m}$. Neurons with these anatomic characteristics have been described previously as both horizontal cells (Germroth et al., 1989) and multipolar cells (Wouterlood et al., 2000). Horizontal cells have been shown to express CCK in the MEC (Schwerdtfeger et al., 1990), whereas layer 1 multipolar cells in the MEC have been described as calretinin (CR)+ (Wouterlood et al., 2000). Both cells have been described as having at least one axonal projection into the deeper layers of the MEC, a feature that was observed in several examples of the cluster 4 neurons. Although Canto and Witter (2012) electrophysiologically characterized layer 1 horizontal and multipolar MEC neurons, their study was focused on principal cells and discarded interneuron-like cells (with shorter AP half-widths) from their analysis. Therefore, to our knowledge, this is the first characterization of these GABAergic, MEC-layer 1-projecting cells. Layer 1 interneurons have been suggested to play a delayed feedback role in cortical computation (Zhou and Hablitz, 1996). Basically, as excitatory inputs arrive from other brain regions and excite pyramidal cells and stellates cells in layers 2 and 3, interneurons in layer 1 may also be excited (either directly by the excitatory inputs or indirectly via principal cells) and inhibit the dendritic branches of the superficial MEC principal cells. Given the larger extentof their axonal projections, it is possible that input to one of these layer 1 interneurons has an effect over a wide area. These may mean inhibitory input onto other layer 1 cells (disinhibition) or inhibitory input onto the dendrites of principal cells in other cortical columns. Further work would be required to understand the specific role these layer 1-projecting cluster 4 neurons play in the MEC.

\section{Previous findings and future directions}

This study emphasizes intrinsic electrophysiological properties and axonal projections in the classification of MEC interneurons. Ferrante et al. (2017) took a complementary approach, analyzing MEC interneurons using several molecular identifiers, including SOM, RCan2, 5HTR3a, and VIP. Like our method, the approach of Ferrante et al. (2017) was effective but included misidentifications; their five electrophysiological parameters together predict biomarker identity with $81 \%$ accuracy. Some of these misidentifications may arise from cells that share molecular markers but have different axonal projection patterns and, conceivably, different electrophysiological properties.

Our clusters correspond only partially with those of Ferrante and colleagues. The PV + interneuron groups in both studies (RCan2 in Ferrante et al., 2017; cluster 2 in this study) were characterized by the lowest input resistance, time constant, and AP half-width and highest peak firing rate when compared to all other interneuron groups. Comparisons with other groups are more difficult. Cluster 1 shares several electrophysiological characteristics with their SOM group, displaying an adaptation ratio of $\sim 0.65$; however our AP half-widths and time constants for cluster 1 are more similar to those of their 5HTR3a interneuron groups. Our cluster 3 was more similar to their SOM group, exhibiting similar AP half-width, adaptation ratio, and time constant characteristics. Cluster 4 does not share an electrophysiological profile with any of the molecular groups in Ferrante et al. (2017). None of the clusters in this study showed as low adaptation ratios as the $5 \mathrm{HTR} 2 \mathrm{a}$ interneuron groups, which suggests that these interneuron types were either not covered in the GAD2 + or PV + cells of this study or are distributed across different clusters. Together, our paper and Ferrante et al. (2017) allow one to make strong predictions of molecular identity and axonal projection pattern based on a rather complete electrophysiological profile, but with some ambiguity and a $10-20 \%$ possibility of a mistaken classification.

Our findings point toward several avenues for future research. For example, the interplay between the slowfiring and fast-firing interneuron populations of layers 2 and 3 is a promising target for understanding the grid cell mechanism. Selective optogenetic manipulation of PV+ and SOM + populations in vivo may help explain how the superficial MEC responds to very heterogeneous inputs and generates grid fields. Anatomic work on the layer 1-projecting cells in this study would explain where this interneuronal population receives inputs (whether mainly from other brain regions or local principal cells) and where its main output targets lie (whether mainly principal cell dendrites or other layer 1 interneurons). Furthermore, spatial variations in interneuronal physiology along the MEC's dorsoventral axis (DVA) could provide vital clues as to the cortical mechanisms behind spatial navigation. Grid field spacing has been shown to increase along the DVA (Hafting et al., 2005). This decrease is matched by a decrease of $\mathrm{PV}+$ inputs and an increase in non-PV + inputs onto the MEC principal cells (Beed et al., 2010), so there exists an inhibitory gradient along the DVA. Given the known spatial correlates along the DVA, uncovering differences in interneuron physiology (for any of the interneuron populations) between the dorsal end interneurons characterized in this study and the unstudied ventral end interneuronal population would be of particular value to the field.

\section{References}

Arthur D, Vassilvitskii S (2007). K-means++: the advantages of careful seeding. Proceedings of the Eighteenth Annual ACM-SIAM Symposium on Discrete Algorithms, pp 1027-1035. Philadelphia: Society for Industrial and Applied Mathematics.

Beed P, Bendels MHK, Wiegand HF, Leibold C, Johenning FW, Schmitz D (2010) Analysis of excitatory microcircuitry in the medial entorhinal cortex reveals cell-type-specific differences. Neuron 68:1059-1066. CrossRef

Beed P, Gundlfinger A, Schneiderbauer S, Song J, Böhm C, Burgalossi A, Brecht M, Vida I, Schmitz D (2013) Inhibitory gradient along the dorsoventral axis in the medial entorhinal cortex. Neuron 79: 1197-1207. CrossRef 
Borg-Graham LJ, Monier C, Frégnac Y (1998) Visual input evokes transient and strong shunting inhibition in visual cortical neurons. Nature 393:369-373.

Buetfering C, Allen K, Monyer H (2014) Parvalbumin interneurons provide grid cell-driven recurrent inhibition in the medial entorhinal cortex. Nat Neurosci 17:710-718. CrossRef

Burak Y, Fiete IR (2009) Accurate path integration in continuous attractor network models of grid cells. PLoS Comput Biol 5:e1000291. CrossRef

Buzsáki G, Geisler C, Henze DA, Wang X-J (2004) Interneuron diversity series: circuit complexity and axon wiring economy of cortical interneurons. Trends Neurosci 27:186-193. CrossRef

Canto CB, Witter MP (2012) Cellular properties of principal neurons in the rat entorhinal cortex. II. The medial entorhinal cortex. Hippocampus 22:1277-1299. CrossRef

Canto CB, Wouterlood FG, Witter MP (2008) What does the anatomical organization of the entorhinal cortex tell us? Neural Plast 2008:1-18.

Cauli B, Porter JT, Tsuzuki K, Lambolez B, Rossier J, Quenet B, Audinat E (2000) Classification of fusiform neocortical interneurons based on unsupervised clustering. Proc Natl Acad Sci USA 97: 6144-6149. CrossRef

Couey JJ, Witoelar A, Zhang SJ, Zheng K, Ye J, Dunn B, Czajkowski R, Moser MB, Moser El, Roudi Y, Witter MP (2013) Recurrent inhibitory circuitry as a mechanism for grid formation. Nat Neurosci 16:318-324. CrossRef

DeFelipe J, López-Cruz PL, Benavides-Piccione R, Bielza C, Larrañaga $P$, Anderson $S$, Burkhalter A, Cauli B, Fairén A, Feldmeyer D, Fishell G, Fitzpatrick D, Freund TF, González-Burgos G, Hestrin S, Hill S, Hof PR, Huang J, Jones EG, Kawaguchi Y, et al. (2013) New insights into the classification and nomenclature of cortical GABAergic interneurons. Nat Rev Neurosci 14:202-216. CrossRef

Domnisoru C, Kinkhabwala AA, Tank DW (2013) Membrane potential dynamics of grid cells. Nature 495:199-204. CrossRef Medline

Dumitriu D, Cossart R, Huang J, Yuste R (2007) Correlation between axonal morphologies and synaptic input kinetics of interneurons from mouse visual cortex. Cereb Cortex 17:81-91. CrossRef

Economo MN, Martínez JJ, White JA (2014) Membrane potentialdependent integration of synaptic inputs in entorhinal stellate neurons. Hippocampus 24:1493-1505. CrossRef

Fernandez FR, Malerba P, White JA (2015) Non-linear membrane properties in entorhinal cortical stellate cells reduce modulation of input-output responses by voltage fluctuations. PLoS Comput Biol 11:e1004188. CrossRef

Ferrante M, Tahvildari B, Duque A, Hadzipasic M, Salkoff D, Zagha EW, Hasselmo ME, McCormick DA (2017) Distinct functional groups emerge from the intrinsic properties of molecularly identified entorhinal interneurons and principal cells. Cereb Cortex 27: 3186-3207.

Fuchs EC, Neitz A, Pinna R, Melzer S, Caputi A, Monyer H (2016) Local and distant input controlling excitation in layer II of the medial entorhinal cortex. Neuron 89:194-208. CrossRef

Garden DLF, Dodson PD, O'Donnell C, White MD, Nolan MF (2008) Tuning of synaptic integration in the medial entorhinal cortex to the organization of grid cell firing fields. Neuron 60:875-889. CrossRef

Gatome CW, Slomianka L, Lipp HP, Amrein I (2010) Number estimates of neuronal phenotypes in layer II of the medial entorhinal cortex of rat and mouse. Neuroscience 170:156-165. CrossRef

Germroth P, Schwerdtfeger WK, Buhl EH (1989) Morphology of identified entorhinal neurons projecting to the hippocampus. A light microscopical study combining retrograde tracing and intracellular injection. Neuroscience 30:683-691. CrossRef

Gloveli T, Schmitz D, Empson R, Dugladze T, Heinemann U (1997) Morphological and electrophysiological characterization of layer III cells of the medial entorhinal cortex of the rat. Neuroscience 77:629-648. CrossRef

Gonzalez-Sulser A, Parthier D, Candela A, McClure C, Pastoll H, Garden D, Surmeli G, Nolan MF (2014) GABAergic projections from the medial septum selectively inhibit interneurons in the medial entorhinal cortex. J Neurosci 34:16739-16743. CrossRef
Hafting T, Fyhn M, Molden S, Moser M-B, Moser El (2005) Microstructure of a spatial map in the entorhinal cortex. Nature 436:801806. CrossRef Medline

Halabisky B (2006) Electrophysiological classification of somatostatinpositive interneurons in mouse sensorimotor cortex. J Neurophysiol 96:834-845. CrossRef

Helm J, Akgul G, Wollmuth LP (2013) Subgroups of parvalbuminexpressing interneurons in layers $2 / 3$ of the visual cortex. J Neurophysiol 109:1600-1613. CrossRef

Hippenmeyer S, Vrieseling E, Sigrist M, Portmann T, Laengle C, Ladle DR, Arber S (2005) A developmental switch in the response of DRG neurons to ETS transcription factor signaling. PLoS Biol 3:e159. CrossRef

Jolliffe IT (2002) Principal component analysis. New York: Springer. Jones RSG, Bühl EH (1993) Basket-like interneurones in layer II of the entorhinal cortex exhibit a powerful NMDA-mediated synaptic excitation. Neurosci Lett 149:35-39. CrossRef

Kepecs A, Fishell G (2014) Interneuron cell types are fit to function. Nature 505:318-326. CrossRef Medline

Krimer LS (2005) Cluster analysis-based physiological classification and morphological properties of inhibitory neurons in layers 2-3 of monkey dorsolateral prefrontal cortex. J Neurophysiol 94:30093022. CrossRef

Kumar SS, Buckmaster PS (2006) Hyperexcitability, interneurons, and loss of GABAergic synapses in entorhinal cortex in a model of temporal lobe epilepsy. J Neurosci 26:4613-4623. CrossRef

Li W-C (2004) A direct comparison of whole cell patch and sharp electrodes by simultaneous recording from single spinal neurons in frog tadpoles. J Neurophysiol 92:380-386. CrossRef

Ma Y (2006) Distinct subtypes of somatostatin-containing neocortical interneurons revealed in transgenic mice. J Neurosci 26:5069_ 5082. CrossRef

Maccaferri G, Lacaille J-C (2003) Interneuron diversity series: hippocampal interneuron classifications: making things as simple as possible, not simpler. Trends Neurosci 26:564-571. CrossRef

MacQueen JB (1967) Some methods for classification and analysis of multivariate observations (Cam LML, Neyman J, eds), pp 281297. Berkeley: University of California Press.

Mancilla JG, Lewis TJ, Pinto DJ, Rinzel J, Connors BW (2007) Synchronization of electrically coupled pairs of inhibitory interneurons in neocortex. J Neurosci 27:2058-2073. CrossRef

McGarry LM (2010) Quantitative classification of somatostatinpositive neocortical interneurons identifies three interneuron subtypes. Front Neural Circuits 4:12.

Miettinen M, Koivisto E, Riekkinen P, Miettinen R (1996) Coexistence of parvalbumin and GABA in nonpyramidal neurons of the rat entorhinal cortex. Brain Res 706:113-122. Medline

Monier C, Chavane F, Baudot P, Graham LJ, Frégnac Y (2003) Orientation and direction selectivity of synaptic inputs in visual cortical neurons. Neuron 37:663-680. CrossRef

Pastoll H, Solanka L, van Rossum MCW, Nolan MF (2013) Feedback inhibition enables theta-nested gamma oscillations and grid firing fields. Neuron 77:141-154. CrossRef

Rousseeuw PJ (1987) Silhouettes: a graphical aid to the interpretation and validation of cluster analysis. J Comput Appl Math 20:5365. CrossRef

Schwerdtfeger WK, Buhl EH, Germroth P (1990) Disynaptic olfactory input to the hippocampus mediated by stellate cells in the entorhinal cortex. J Comp Neurol 292:163-177. CrossRef Medline

Shipston-Sharman O, Solanka L, Nolan MF (2016) Continuous attractor network models of grid cell firing based on excitatoryinhibitory interactions: excitatory-inhibitory grid networks. J Physiol 594:6547-6557. CrossRef

Sokal RR (1958) A statistical method for evaluating systematic relationships. Univ Kans Sci Bull 38:1409-1438.

Solanka L, van Rossum MC, Nolan MF (2015) Noise promotes independent control of gamma oscillations and grid firing within recurrent attractor networks. Elife 4. CrossRef 
Soriano E, Martinez A, Farińas I, Frotscher M (1993) Chandelier cells in the hippocampal formation of the rat: the entorhinal area and subicular complex. J Comp Neurol 337:151-167. CrossRef

Tahvildari B, Alonso A (2005) Morphological and electrophysiological properties of lateral entorhinal cortex layers II and III principal neurons. J Comp Neurol 491:123-140. CrossRef

Taniguchi H, He M, Kim S, Paik R, Sugino K, Fu Y, Lu J, Lin Y, Miyoshi G, Shima Y, Fishell G, Nelson SB, Huang ZJ (2011) A resource of Cre driver lines for genetic targeting of GABAergic neurons in cerebral cortex. Neuron 71(6):995-1013. CrossRef

Thurley K, Hellmundt F, Leibold C (2013) Phase precession of grid cells in a network model without external pacemaker. Hippocampus 23:786-796.

Varga C, Lee SY, Soltesz I (2010) Target-selective GABAergic control of entorhinal cortex output. Nat Neurosci 13:822-824. CrossRef Medline

Wehr M, Zador AM (2003) Balanced inhibition underlies tuning and sharpens spike timing in auditory cortex. Nature 426:442-446. CrossRef

Whittington MA, Traub RD (2003) Interneuron diversity series: inhibitory interneurons and network oscillations in vitro. Trends Neurosci 26:676-682. CrossRef

Wolansky T, Pagliardini S, Greer JJ, Dickson CT (2007) Immunohistochemical characterization of substance $P$ receptor $(N K(1) R)$ - expressing interneurons in the entorhinal cortex. J Comp Neurol 502:427-441. CrossRef

Woodhall GL, Bailey SJ, Thompson SE, Evans DIP, Jones RSG (2005) Fundamental differences in spontaneous synaptic inhibition between deep and superficial layers of the rat entorhinal cortex. Hippocampus 15:232-245. CrossRef

Wouterlood FG, Pothuizen H (2000) Sparse colocalization of somatostatin- and GABA-immunoreactivity in the entorhinal cortex of the rat. Hippocampus 10:77-86. CrossRef Medline

Wouterlood FG, Härtig W, Brückner G, Witter MP (1995) ParvaIbumin-immunoreactive neurons in the entorhinal cortex of the rat: localization, morphology, connectivity and ultrastructure. J Neurocytol 24:135-153. CrossRef

Wouterlood FG, van Denderen JC, van Haeften T, Witter MP (2000) Calretinin in the entorhinal cortex of the rat: distribution, morphology, ultrastructure of neurons, and co-localization with gammaaminobutyric acid and parvalbumin. J Comp Neurol 425:177-192. CrossRef

Yekhlef L, Breschi GL, Lagostena L, Russo G, Taverna S (2015) Selective activation of parvalbumin- or somatostatin-expressing interneurons triggers epileptic seizurelike activity in mouse medial entorhinal cortex. J Neurophysiol 113:1616-1630. CrossRef Medline

Zhou F-M, Hablitz JJ (1996) Morphological properties of intracellularly labeled layer I neurons in rat neocortex. J Comp Neurol 376:198-213. CrossRef 